\title{
Prolonged partner separation erodes nucleus accumbens transcriptional signatures of pair bonding in male prairie voles
}

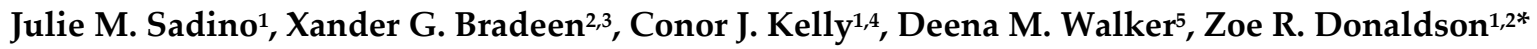 \\ ${ }^{1}$ Department of Molecular, Cellular, and Developmental Biology, University of Colorado Boulder, Boulder, CO 80309 \\ 2 Department of Psychology and Neuroscience, University of Colorado Boulder, Boulder, CO 80309 \\ ${ }^{3}$ Department of Adult Hematology, University of Colorado- Anschutz Medical Campus, Aurora, CO 80045 \\ ${ }^{4}$ BioFrontiers Institute, University of Colorado Boulder, Boulder CO 80303 \\ ${ }^{5}$ Department of Behavioral Neuroscience, Oregon Health and Science University, School of Medicine, Portland, OR \\ 97239 \\ * Correspondence: Email: zoe.donaldson@colorado.edu
}

\begin{abstract}
$\underline{\text { Abstract }}$
The loss of a spouse is often cited as the most traumatic event in a person's life. However, for most people, the severity of grief and its maladaptive effects subside over time via an understudied adaptive process. Like humans, socially monogamous prairie voles (Microtus ochrogaster) form opposite-sex pair bonds, and upon partner separation, show behavioral and neuroendocrine stress phenotypes that diminish over time. Eventually, they can form a new bond, a key indicator of adapting to the loss of their partner. Thus, prairie voles provide an ethologically-relevant model for examining neuromolecular changes that emerge following partner separation for adapting to loss. Here, we test the hypothesis that extended partner separation diminishes pair bond-associated behaviors (partner preference and selective aggression) and causes pair bond transcriptional signatures to erode. Pairs were cohoused for 2 weeks and then either remained paired or were separated for $48 \mathrm{hrs}$ or $4 \mathrm{wks}$ before collecting fresh nucleus accumbens tissue for RNAseq. In a separate cohort, we assessed partner preference and selective aggression at these time points. Surprisingly, pair bond-associated behaviors persist despite prolonged separation and are similar between same-sex and opposite-sex paired voles. In contrast, we found that opposite-sex pair bonding, as compared with same-sex pairing, led to changes in accumbal transcription that were stably maintained as long as animals remained paired but eroded following prolonged partner separation. Eroded genes are primarily associated with gliogenesis and myelination, suggesting a previously undescribed role for glia in maintaining pair bonds and adapting to partner loss. We further reasoned that relevant neuronal transcriptional changes may have been masked by the prominent transcriptional signals associated with glia. Thus, we pioneered neuron-specific translating ribosomal affinity purification in voles. Neuronallyenriched transcriptional changes revealed dopaminergic-, mitochondrial-, and steroid hormone signalingassociated gene clusters whose expression patterns are sensitive to acute pair bond disruption and loss adaptation. Together, our results suggest that partner separation results in erosion of transcriptomic signatures of pair bonding despite core behavioral features of the bond remaining intact, revealing potential molecular processes central to priming a vole to be able to form a new bond.
\end{abstract}

Keywords: prairie vole, loss, pair bond, nucleus accumbens

\section{Introduction}

The death of a romantic partner is cited as one of the most traumatic experiences in a person's life $(1,2)$. However, the majority of people will adapt to loss, reengage with life, and will eventually form a new pair bond (3). The processes that enable adapting to partner loss remain poorly understood but likely occur via the same neural systems that are uniquely engaged by pair bonding (4).

Socially monogamous prairie voles are a laboratory-amenable species that recapitulates many aspects of human social bonds, making them ideal for 
Sadino et al., 13 July $2021-$ BioRxiv

interrogating the neurobiology of bonding and loss. Pair bonded prairie voles prefer to spend time with their partner compared with other voles, show robust biparental care, and exhibit distress upon partner separation (5-15). In addition, we have previously shown that if a pair-bonded prairie vole loses their partner, given enough time, they will form a new bond that supersedes the original (16). The ability to form a new bond following prolonged partner separation provides a behavioral indicator of loss adaptation.

Comparison of opposite-sex and same-sex prairie vole pairs provides a powerful means to identify the neurobiological changes specific to pair bonding independent of those that support affiliative relationships more generally $(4,17-19)$. Specifically, pair bonds, when compared to same-sex pairs, induce altered neuropeptide signaling and receptor expression throughout the brain (10, 20-24). In both sexes, formation of a pair bond yields a robust change in transcription in the nucleus accumbens (NAc), a brain region extensively implicated in pair bonding and loss adaptation $(20,21)$. Such pair bondinduced transcriptional changes may solidify the exclusive rewarding aspects of a bond, and by this logic, it is likely that transcriptional changes in the NAc are also important for adapting to partner loss.

We compared male voles separated from an opposite-sex pair bonded partner or same-sex peer to identify transcriptional changes specific to partner loss independent of the effects of general social isolation or loss of other affiliative relationships. We hypothesized that pair bond transcriptional signatures and bond-associated behaviors would erode following prolonged partner separation. We reasoned that these changes represent key components of loss adaptation that, together, may prime the vole to be able to form a new bond. We found that pairing induces a reliable affiliative preference for a peer or a pair bonded partner, and that this preference is remarkably stable, persisting even after four weeks of separation. We further show that pair-bond associated changes in accumbal transcription remain consistent from two to six weeks post-pairing. However, once opposite-sex pairs are separated, the pair bond transcriptional signature erodes as a function of separation time. To further hone in on the transcriptional changes associated with partner separation specifically in NAc neurons, we pioneered translating ribosomal affinity purification in voles (vTRAP). Using VTRAP, we identified clusters of genes associated with dopaminergic signaling, mitochondrial organization, and steroid hormone signaling whose expression patterns are sensitive to acute pair bond disruption and loss adaptation. In sum, our behavioral and transcriptional data suggests that erosion of pair bond transcriptional signatures in the NAc precedes changes in affiliative partner preference and selective aggression, providing insight into time-dependent neuromolecular changes that may contribute to loss adaptation.

\section{$\underline{\text { Results }}$}

We determined how extended social separation affects hallmark behaviors of pair bonds in male voles in opposite-sex $(n=16)$ and same-sex $(n=15)$ pairs. Fig. 1A shows the within-animal experimental time course used to test each animal's partner preference and selective aggression after short-term (48 hours) and long-term (4 weeks) separation. All sample sizes and comprehensive statistical results, including effect size estimates, are reported in Supplementary Table 1.

\section{Extended separation does not reduce partner preference}

We hypothesized that opposite-sex pairs would have a partner preference at baseline and after short-term separation, when the pair bond is still intact, but not after long-term separation when the pair bond is ostensibly weakened (16). Similarly, we anticipated that same-sex pairs would have a partner preference at baseline but would exhibit a faster and more robust loss of partner preference when separated. Both opposite-sex and samesex pairs form a selective preference for their partner after 2 weeks of cohabitation (one sample t-test relative to $50 \%$ : opposite-sex $\mathrm{p}=0.00015$, same-sex $\mathrm{p}=0.064$ ) and retain their partner preference after short-term and long-term separation (one sample t-test relative to $50 \%$ : opposite-sex short-term $p=0.00023$, opposite-sex long-term $p=0.0016$; same-sex short-term $p=0.050$, same-sex long-term $p=$ 0.0037). Additionally, there was no significant difference in partner preference score between opposite-sex and samesex pairs over time (Fig. 1B; 2-way RM-ANOVA: Main effect of partner (opposite- vs same-sex): $\mathrm{F}_{(1,84)}=1.40, \mathrm{p}=$ $0.24, \eta=0.016$; Main effect of time: $\mathrm{F}_{(2,84)}=0.18, \mathrm{p}=0.83, \eta=$ 0.0043; Partner $X$ time: $\left.\mathrm{F}_{(2,84)}=0.12, \mathrm{p}=0.89, \eta=0.0028\right)$. Other commonly used metrics, including time spent huddling and distance from either conspecific, also did not show significant differences between groups, over time, or as an interaction of these variables (Fig. S1A-C; Table S1). Together, these results indicate that prairie voles are 
bioRxiv preprint doi: https://doi.org/10.1101/2021.07.14.452355; this version posted July 14, 2021. The copyright holder for this preprint (which was not certified by peer review) is the author/funder, who has granted bioRxiv a license to display the preprint in perpetuity. It is made available under aCC-BY-NC-ND 4.0 International license.

Sadino et al., 13 July 2021 -BioRxiv

A

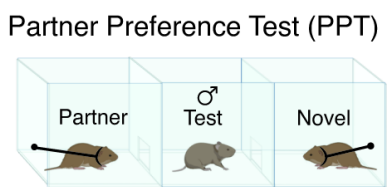

Resident Intruder Test (RIT)

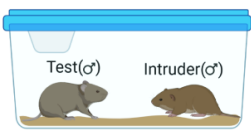

B

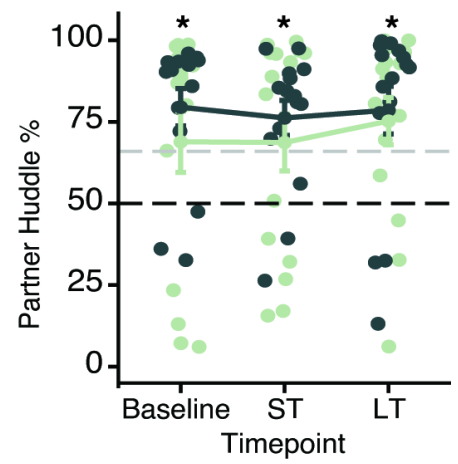

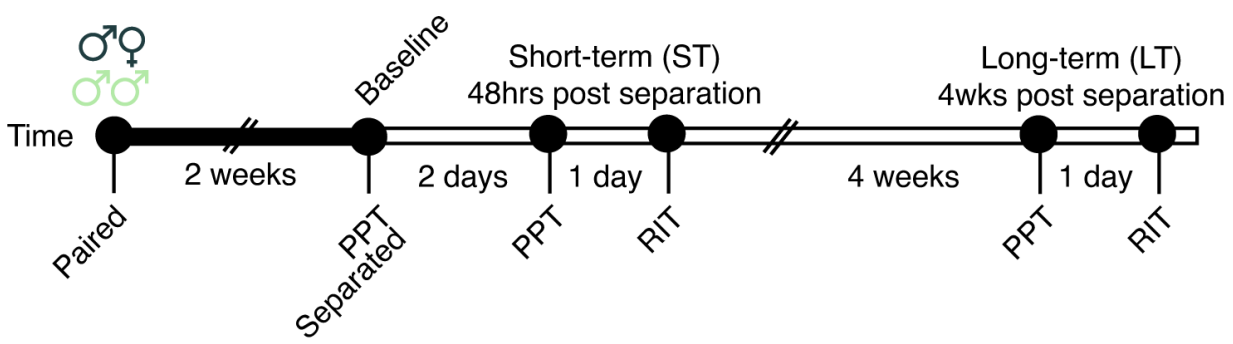

PairingType Opposite-Sex Same-Sex

C

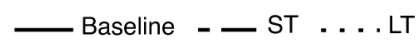

D

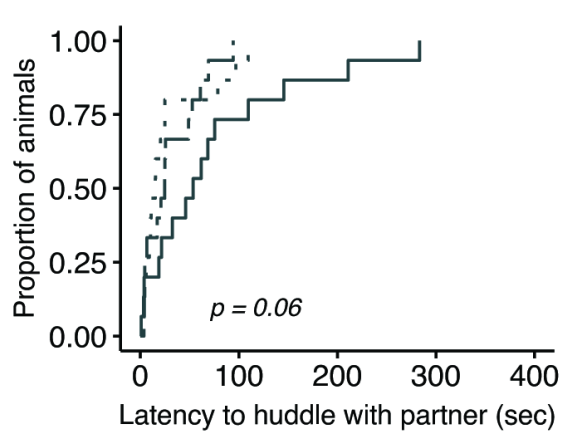

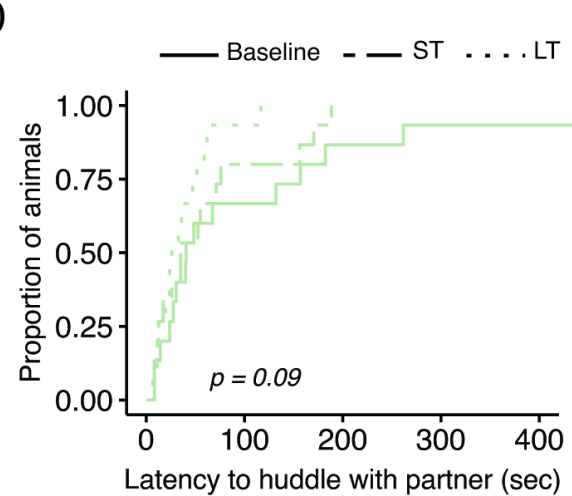

E

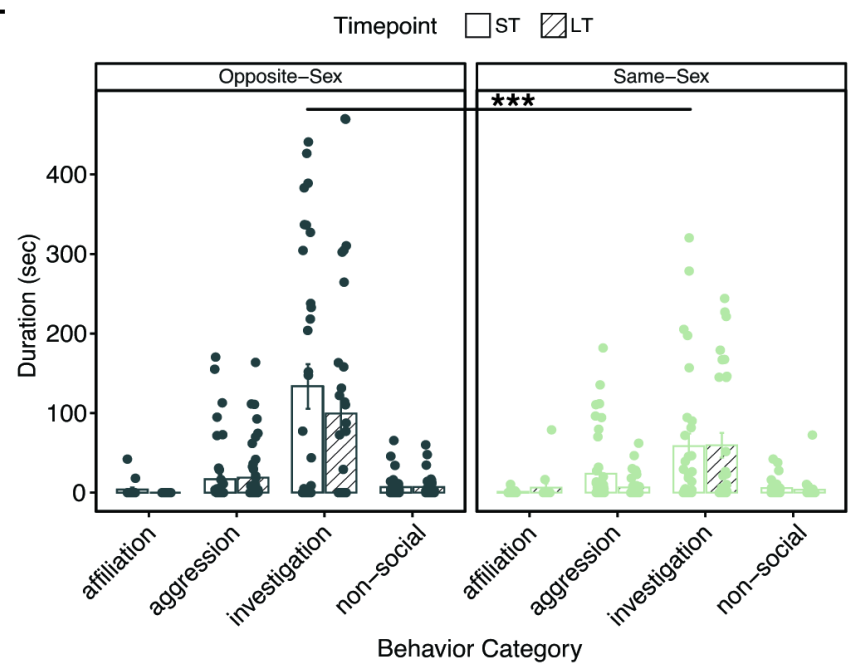

$\mathrm{F}$

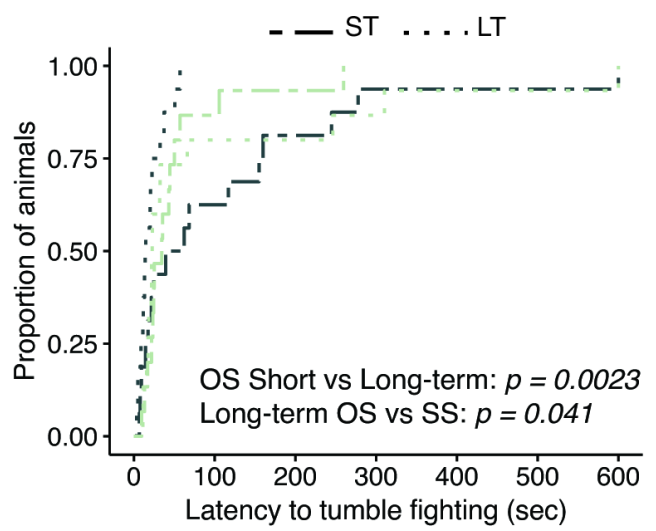

Fig. 1 Males in opposite-sex pairs retain their partner preference and selective aggression for at least four weeks following partner separation. (A) Schematics of behavioral tests and timeline for behavioral experiments. Opposite-sex (OS; $n=16)$ and same-sex (SS; $n=15)$ pairs were paired for 2 weeks prior to a baseline partner preference test (PPT). Pairs were then separated for 48 hrs prior to short-term (ST) behavior tests of PPT and resident intruder (RIT) administered $24 \mathrm{hrs}$ apart. Animals remained separated until the same behavior tests were repeated 4 wks later for the long-term (LT) time point. (B) Partner preference scores (\% partner huddle/total huddle) from baseline, short-term, and long-term PPTs of opposite-sex (dark green) and same-sex (light green) paired males. Opposite- and same-sex paired males showed a baseline partner preference (one way t-test relative to $50 \%$; opposite-sex: $\mathrm{T}_{15}=5.14$, $\mathrm{p}<0.001$, same-sex: $\left.\mathrm{T}_{15}=2.014, \mathrm{p}=0.064\right)$ that remained evident for both groups after short-term separation (opposite-sex: $\mathrm{T}_{15}=3.908, \mathrm{p}<0.001$, samesex: $\mathrm{T}_{15}=2.149, \mathrm{p}=0.050$ ) and long-term separation (opposite-sex: $\mathrm{T}_{15}=3.908, \mathrm{p}<0.002$, same-sex: $\mathrm{T}_{15}=3.477, \mathrm{p}=0.004$ ). Black dotted line at 50\% indicates no preference and gray dotted line indicates $2 / 3$ of time with partner. (C, D) Cumulative event plots indicating that opposite-sex males huddle with their partner more quickly after separation $\left(\mathrm{C}: \log\right.$-rank test: $\left.\chi^{2}=5.6, \mathrm{p}=0.06\right)$ while same-sex males show minimal changes in partner huddle latency (D: log-rank test: $\left.\chi^{2}=4.9, \mathrm{p}=0.087\right)$. (E) Bar graph of the duration (sec) of each behavior in the resident-intruder test for opposite-sex (left) and same-sex (right) after short-term (solid box) and long-term (hatched box) separation. There is a significant difference in social investigation between opposite- and same-sex paired males at the short-term time point (3-way RM-ANOVA with Tukey's post-hoc: $p=0.00097)$ but no other differences were observed. (F) Cumulative event plot showing latency to tumble fighting $(\mathrm{sec})$ in the resident intruder test. After long-term separation, opposite-sex males were significantly faster to engage in tumble fighting, a marker of intense aggression, compared to the short-term time point $(\log$-rank test: $\chi 2=9.30, p=0.0023)$ or when compared to same-sex paired males (log-rank test: $\chi 2=4.20, \mathrm{p}=0.041)$. 
Sadino et al., 13 July 2021-BioRxiv

capable of forming an affiliative preference for an oppositeor same-sex partner that remains intact despite prolonged separation.

\section{Subtle differences in opposite-sex and same-sex partner preference are evident in locomotion, latency to huddle, and behavioral consistency across tests}

As none of the typically employed partner preference test metrics indicated pairing-type-dependent differences, we investigated more subtle behavioral phenotypes. We first asked if there were differences in locomotion or partner huddle latency to query if oppositeor same-sex paired animals spent more time investigating their social environment. We reasoned that opposite-sexpaired animals would be more vigilant in assessing novel animals and would be more motivated to interact with their partner than same-sex-paired animals. Opposite-sexseparated males had significantly greater locomotion at baseline and after short-term separation but were indistinguishable from same-sex paired voles after longterm separation (Fig. S1D, 2-way RM-ANOVA with Tukey's post hoc: Baseline opposite- vs same-sex $p=0.0017$, short-term opposite- vs same-sex $p=0.024$, long-term opposite- vs same-sex $p=0.36$ ). This increased locomotion may reflect increased vigilance in pair bonded males in a new environment. Opposite-sex males also began huddling with their partner faster over the course of separation while there were no differences in latency to huddle with a novel animal in either opposite-sex or same-sex males (Fig. 1C, D; Table S1, log-rank test main effect of time points: oppositesex partner $x^{2}=5.6, p=0.06$, novel $x^{2}=0.60, p=0.72$; samesex partner $x^{2}=4.90, p=0.087$, novel $x^{2}=1.30, p=0.52$; interaction time $X$ partner (opposite- v same-sex) $X^{2}=13.90$, $\mathrm{p}=0.02$; interaction time $X$ novel (opposite- $v$ same-sex) $X^{2}=$ $2.40, p=0.80$ ). This decrease in huddle latency on oppositesex paired males may reflect enhanced motivation to be with their absent partner.

We next examined behavioral consistency across partner preference tests. First, we examined the consistency in partner preference scores between time points in opposite-sex and same-sex groups. The partner preference change score was calculated by subtracting the percent partner preference between time points (short-term separation - baseline; long-term separation - baseline) (Fig. S1E). Following short-term separation, opposite-sexseparated animals showed more consistent partner preference scores than same-sex animals while long-term separation caused the opposite-sex and same-sex groups to be indistinguishable from each other (Fig. S1E; F-test of equality of variances: short-term:baseline $\mathrm{F}_{(14)}=0.21, \mathrm{p}=$ 0.0054; long-term:Baseline $\left.\mathrm{F}_{(14)}=0.65, \mathrm{p}=0.43\right)$. Second, we examined the consistency of pair bond behaviors as a function of separation time by assessing the strength of correlations between individual behaviors from baseline to short-term and baseline to long-term in opposite-sex or same-sex separated animals (partner and novel huddle times, total huddle time, partner preference score, and locomotion) (Fig. S1F). Strong social behavioral correlations were observed in opposite-sex paired animals between baseline and short-term separation but not after long-term separation, suggesting that pair bond behaviors destabilize following extended separation.

Same-sex paired animals showed uniformly weaker correlations across all timepoints. However, locomotor behavior remained correlated across all timepoints for opposite- and same-sex voles, indicating long-term separation selectively destabilizes social behaviors.

\section{Long-term separation reduces selective aggression in same- sex but not opposite-sex paired voles}

As has been reported previously, both oppositeand same-sex-paired males demonstrated aggression towards a novel male vole in a resident intruder test (17). We hypothesized that opposite-sex-paired animals would be aggressive after short-term separation, when the pair bond is still intact, but not after long-term separation, when the pair bond is weakened. In same-sex animals, we anticipated reduced aggression after long-term separation and overall less aggression than opposite-sex-paired males. We found that aggressive behavior did not differ between pairing types after short-term separation (2-way RMANOVA in Table S1). Only same-sex-separated animals showed a reduction in tumble fighting duration and total aggression behaviors (summed duration of tumble Fighting, chasing and defensive postures) between shortterm and long-term separation (Fig. 1E, Fig. S1G stats Table S1). As a second metric, we also examined the latency to begin tumble fighting. Opposite-sex males were both faster to begin tumble fighting following prolonged separation and were faster to aggress compared to same-sex males after long-term separation (Fig. 1F, log-rank test: opposite-sex latency (short- vs long-term) $x^{2}=9.30, p=$ 0.0023; (long-term opposite- vs same-sex) $\mathrm{x}^{2}=4.20, \mathrm{p}=0.04$ ). 
Affiliative behaviors (huddling) and non-social behaviors (autogrooming and digging) did not differ significantly either by partner type or separation time (Fig. 1E, Table S1).

\section{The pair bond transcriptional signature is stable over time}

Changes in many behavioral states are supported by stable changes in transcription, although this has not been examined in the context of selective social bonds (2528). Specifically, while prior work has demonstrated that mating and cohabitation result in transcriptional changes within the NAc, the consistency of these changes as long as the bond remains intact has yet to be assessed (20-22, 29, 30). Thus, we compared NAc transcription in oppositeversus same-sex-paired voles following either 2 or 6 weeks of pairing/cohabitation (Fig. 2B). By comparing oppositesex to same-sex-paired animals, we identified transcripts specific to pair bonds compared with those associated with affiliative behavior more generally. For consistency, we limited transcriptional assessment to voles that had a baseline partner preference $>50 \%$ (Fig. 2B; Table S1).

We used DESeq2 to determine differential gene expression in opposite- relative to same-sex paired males after short-term and long-term pairing (Fig. 2D) (31). Global transcriptional differences for opposite- versus same-sex pairs were strikingly similar following either 2 or 6 weeks of pairing, suggesting stable pair-bond associated transcription across these timepoints (Fig. 2F). We next identified differentially expressed genes (DEGs) using $\log _{2}$ FoldChange $>0.30$ or $<-0.30$ with a $p$-value $>0.05$ based on prior work showing that such differences are replicable and detectable via alternate methods (32) (DEGs are listed in Table S2). While we identified more DEGs following 6 weeks of pairing, there was significant overlap in the upregulated (104 shared, Fisher's Exact Test: $x^{2}=30.24, p=$ $5.36 \times 10^{-31}$ ) and downregulated (46 shared, Fisher's Exact Test: $\left.X^{2}=13.32, p=7.05 \times 10^{-14}\right)$ transcripts between timepoints (Fig. 2E) (33).

To further examine similarity in transcriptional patterns between short-term and long-term pair bonds we employ Rank-Rank Hypergeometric Overlap (RRHO) (Fig. 2G) $(34,35)$. This threshold free approach allows us to determine if similar global transcription are observed between two comparisons. The resulting RRHO heatmap is arranged into quadrants based on the direction of gene expression and each point represents the significance derived from the number of overlapping genes via the hypergeometric distribution (Fig. 2G). We compared the opposite- vs same-sex 2-week paired to opposite-sex vs same-sex 6-week paired differential gene expression data to determine how similar global transcription is between short-term and long-term pair bonds. We observed extensive concordance of transcriptional patterns between 2- and 6-week paired voles, further supporting that the transcriptional profile of pair bonds is stable (Fig. $2 \mathbf{H}$ ).

Based on the similarity of the pair bond transcriptional profiles over time, the $\sim 2$ week and 6 week animals from each pairing type were pooled to create a single opposite-sex- vs same-sex-paired comparison. We refer to the time-pooled opposite-sex vs same-sex comparison as the combined pair bond transcriptional signature. By doing so, we create a single, well powered group describing pair bond transcription against which we can assess changes in transcription resulting from partner separation (Fig. 2I).

To determine how biological pathways might be influencing the pair bond transcriptional profile we employed Gene Ontology (GO) and Ingenuity Pathway Analysis (IPA) (36). GO terms associated with upregulated DEGs of the combined pair bond and the quadrant UU genes strongly implicate changes in glial cells and extracellular matrix organization (Fig. 2J), which is mirrored by activation of glioblastoma signaling via IPA (Fig. S3A). IPA additionally identified upregulation of Synaptogenesis, CREB signaling, and Endocannabinoid Neuronal Synapse pathways. All of these pathways are consistent with pair bonding as a form of complex learning that is mediated by neuromodulatory signaling (37-40). Predicted upstream regulators of these pathways broadly support a role for learning and neuromodulation, including Creb1, Esr1, and various growth-factor and developmental genes (Fig. S3A). GO terms associated with downregulated DEGs of the combined pair bond and the quadrant DD genes are implicated in nucleoside synthesis and synaptic plasticity (Fig. 2J). IPA indicates a suppression of corticotropin releasing hormone signaling, potentially reflecting the strong social buffering of stressors that occurs in pair bonded voles specifically $(13,41-44)$ (Fig. S3A). Finally, the strong correspondence in the significance of GO terms between the combined pair bond DEGs and the RRHO quadrants further validates that pooling the time points retains biologically relevant transcriptional signatures (Fig. 2J). 
bioRxiv preprint doi: https://doi.org/10.1101/2021.07.14.452355; this version posted July 14, 2021. The copyright holder for this preprint (which was not certified by peer review) is the author/funder, who has granted bioRxiv a license to display the preprint in perpetuity. It is made available under aCC-BY-NC-ND 4.0 International license.

Sadino et al., 13 July 2021-BioRxiv

A

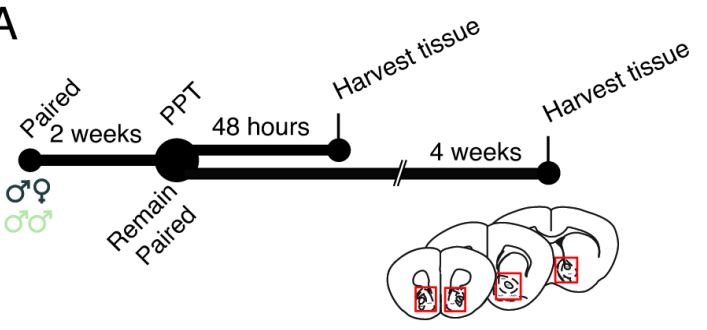

B

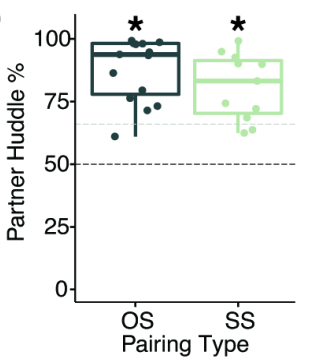

$\mathrm{C}$

\begin{tabular}{|c|c|}
\hline $\begin{array}{l}\text { g } \\
\stackrel{心}{u ̈ ~}\end{array}$ & $\begin{array}{l}\text { Pair bond defined as OS expression relative to SS at the } \\
\text { short and long-term } \\
\text { Compare DEGs and global transcriptional expression of } \\
\text { short and long-term pair bond }\end{array}$ \\
\hline 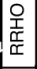 & $\begin{array}{l}\text { Transform DESeq gene lists to confirm genome wide } \\
\text { similarities in pair bond signatures }\end{array}$ \\
\hline 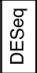 & $\begin{array}{l}\text { Combine short and long-term groups to define a } \\
\text { combined pair bond signature }\end{array}$ \\
\hline 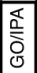 & Determine enriched biological pathways of a stable pair bond \\
\hline
\end{tabular}

$\mathrm{E}$

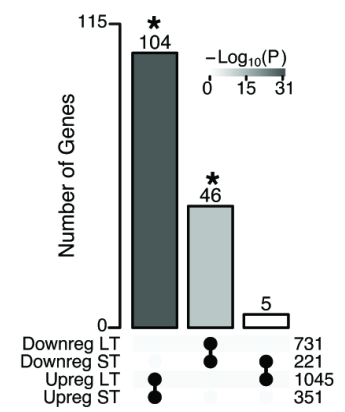

Upreg LT

51

$\mathrm{F}$
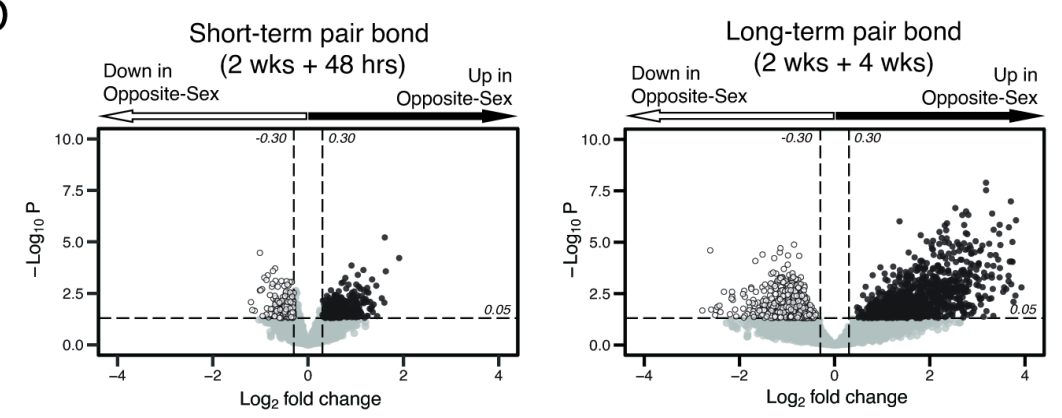

Long-term pair bond

\section{ST pair bond \\ LT pair bond}

G

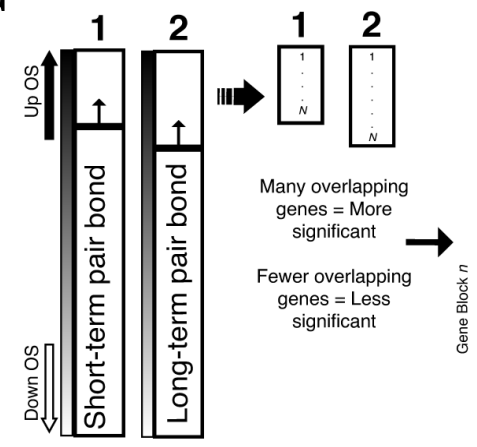

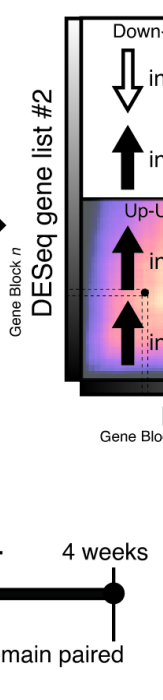

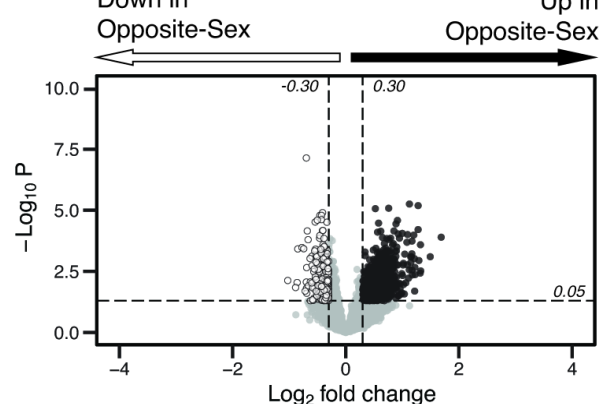

scaled $\log _{2}$ FoldChange
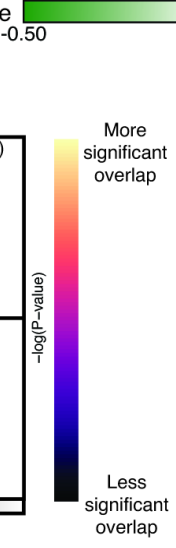

$\mathrm{H}$

H Concordant pair bond gene expression over time
Combined Pair Bond

Down in

I

in DESeq gene list \#1

$J$

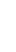

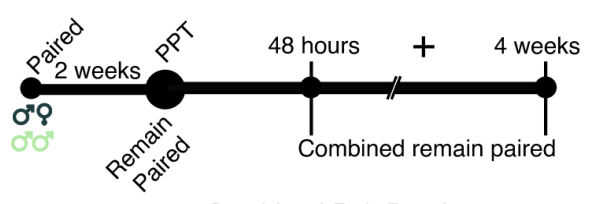

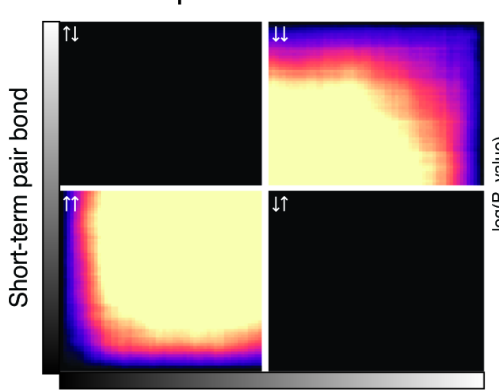

Long-term pair bond

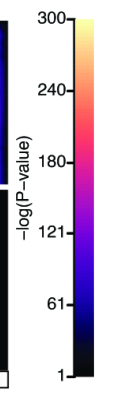

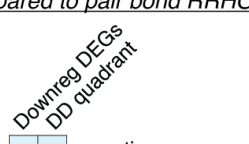

exocytic process

purine-containing compound metabolic process

purine ribonucleoside triphosphate metabolic process nucleoside triphosphate metabolic process nucleoside phosphate biosynthetic process

GTP metabolic process

nucleotide metabolic process

vesicle docking

synapse organization

vesicle docking involved in exocytosis

Golgi organization

negative regulation of cell projection organization

organelle localization by membrane tethering

membrane docking

regulation of synapse organization 
Fig. 2 Pair bonding leads to persistent and consistent changes in NAc transcription. (A) Opposite- and same-sex pairs were paired for 2 weeks prior to a baseline partner preference test. Pairs then remain paired for either 48 hours (short-term; $\sim 2$ weeks total pairing) or 4 weeks (long-term; $\sim 6$ weeks total pairing) prior to collecting fresh nucleus accumbens tissue (dissection sites in red boxes) for RNA sequencing. (B) Baseline partner preference scores of males included in RNA sequencing for the opposite- and same-sex groups (one-tailed t-test relative to $50 \%$ : opposite-sex $\mathrm{T}_{14}=11.76$, $\mathrm{p}=1.21 \mathrm{X} 10^{-8}$; same-sex $\mathrm{T}_{10}=7.78, \mathrm{p}=1.50 \times 10^{-5}$ ). Black dotted line indicates a $50 \%$ partner preference score and the grey dotted line indicates $66 \%$. There were no differences in partner preference score between opposite- and same-sex paired animals used for RNAseq (two-tailed t-test: $\left.\mathrm{T}_{21.016}=1.374, \mathrm{p}=0.184\right)$. $(\mathbf{C})$ Transcriptional analysis workflow. (D) Differential gene expression using DESeq2 where genes upregulated in opposite-sex are to the right (black dots) and genes downregulated in opposite-sex are to the left (white dots). (E) Upset plot showing overlap of up- and downregulated DEGs from each timepoint. Intersections with no overlapping genes, such as downregulated long-term:upregulated short-term, are not shown. Asterisks and shading indicate statistically significant intersections (Fisher's Exact Test: Upreg ST:LT $\chi^{2}=30.24, p=5.36$ X 10-31; Downreg ST:LT $\chi^{2}=13.32$, $p=7.05$ X $10^{-14}$ ). (F) Every gene from short-term and long-term pairing ordered from the smallest to largest $\log _{2}$ FoldChange after short-term pairing. (G) Schematic of RRHO analysis. The heatmap is arranged into quadrants of genes upregulated in both lists (up-up: quadrant UU), downregulated in both lists (down-down: quadrant DD), or genes that have opposite regulation (up in list 1-down in list 2: quadrant UD; down in list 1-up in list 2: quadrant DU). Genes that are found in both lists at a similar ranked position result in higher p-values and are represented here by a yellow color. (H) RRHO comparing short-term and long-term pair bonding (from $2 D$ ) indicates a stable pair bond gene signature over time as evidenced by concordant up- or downregulated genes at the two timepoints. (I) The shortterm and long-term time points were pooled for opposite- and same-sex pairs to define the combined pair bond gene signature. (J) We compared GO analysis Mus musculus ontology terms between the combined pair bond DEGs and the RRHO quadrants (from $2 \mathrm{H}$ ). Strong correspondence in the GO term significance between the two analyses supports that the combined pair bond transcriptional signature retains relevant biological information.

\section{The pair bond transcriptional signature erodes following prolonged partner separation}

We next tested the hypothesis that the pair bond transcriptional signature erodes following long-term partner separation. We paired opposite-sex and same-sex pairs for 2 weeks prior to a baseline partner preference test and then immediately separated males into new cages for either 48 hours (short-term) or 4 weeks (long-term) prior to harvesting NAc tissue for RNAseq (Fig. 3A). As before, we only included animals with a baseline partner preference $>50 \%$ (Fig. 3B). We determined the effect of separation on the pair bond gene signature by first defining differential gene expression of opposite- vs same-sex animals after short- and long-term separation and then used RRHO to examine genome-wide expression changes relative to the combined pair bond signature (Fig. 3C).

First, we performed differential expression analysis of opposite- vs same-sex-separated males at each separation time point (Fig. 3D). There were significantly more shared DEGs between the combined pair bond and short-term separation than would be expected by chance, but this was not observed when comparing the shared genes between the combined pair bond and long-term separation (Fig. 3E, Fisher's exact test: pair-bond:short-term sep $x^{2}=31.86, p=$ $6.78 \times 10^{-19}$; pair-bond:long-term sep $x^{2}=17.96, p=0.53$ ). Further, there are even fewer shared DEGs between shortand long-term separation suggesting notable transcriptional differences between these time points (Fig. 3E). The difference in transcription between short- and long-term separation is particularly evident when comparing the global transcriptomes, which display similarity between the combined pair bond and short-term separation, and large dissimilarity following long-term separation (Fig. 3F). Together, these results suggest that extended separation erodes nucleus accumbens pair bond transcription, a potentially crucial molecular process needed to prime the vole to be able to form a new bond.

To more comprehensively assess genome-wide changes to the pair bond signature following extended partner separation we again employed RRHO (Fig. 3G). We compared the ranked gene list of opposite- vs same-sex for the 1) combined pair bond gene signature (Fig. 2G) versus either 2) short-term partner separation (Fig. 3D, left) or 3) long-term partner separation (Fig. 3D, right). We found that the pair bond signature remains largely intact after short-term separation as indicated by significant signal among upregulated genes (quadrant UU), and to a lesser extent, downregulated genes (quadrant DD) (Fig. 3G). However, following long-term separation, the pair bond gene signature is largely undetectable as indicated by a lack of significant signal throughout the UU and DD quadrants of the RRHO plot. Plotting the long-term RRHO with an unadjusted $p$-value scale revealed few overlapping genes in the UU and DD quadrants. The low level of overlap indicates that there is a residual, albeit greatly reduced, pair bond signature that is still detectable after long-term separation (Fig. S2C). As a further control, we randomly shuffled gene ranking of the combined pair bond genes which ablated the RRHO signal observed in Fig. $2 \mathrm{H}$ and Fig. 3G (Fig. S2G-I).

We next examined which genes are consistently present in the UU or DD quadrants across pair bonding and separation. Specifically, we compared the UU and DD quadrants (inclusive of all transcripts in each quadrant) of the short-term (2 week) vs long-term (6 week) pair bond from Fig. $2 \mathrm{H}$, the combined pair bond vs short-term separation in the left side of Fig. 3G, and the combined pair bond vs long-term separation on the right side of Fig. 3G. The degree of overlap in the UU quadrants was greatest between the combined pair bond vs short-term separation 
bioRxiv preprint doi: https://doi.org/10.1101/2021.07.14.452355; this version posted July 14, 2021. The copyright holder for this preprint (which was not certified by peer review) is the author/funder, who has granted bioRxiv a license to display the preprint in perpetuity. It is made available under aCC-BY-NC-ND 4.0 International license.

Sadino et al., 13 July $2021-$ BioRxiv

A

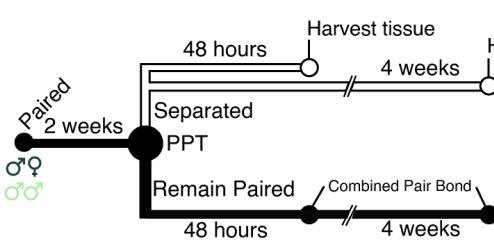

B

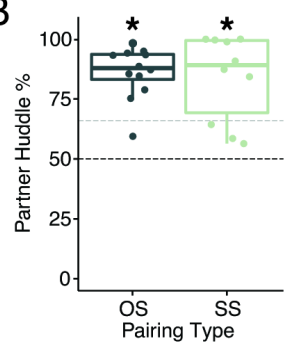

C

\begin{tabular}{|c|c|}
\hline 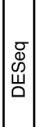 & $\begin{array}{l}\text { Changes to pair bond defined as OS expression relative to } \\
\text { SS after short and long-term separation } \\
\text { Compare DEGs and global transcriptional expression of } \\
\text { the combined pair bond to short and long-term separation }\end{array}$ \\
\hline & $\begin{array}{l}\text { Transform DESeq gene lists to identify genome wide } \\
\text { changes to the pair bond signature after separation } \\
\text { Compare the RRHO quadrants of the combined pair bond } \\
\text { to short and long-term separation }\end{array}$ \\
\hline 全 & $\begin{array}{l}\text { Determine enriched biological pathways of genes } \\
\text { resistent and susceptible to separation }\end{array}$ \\
\hline
\end{tabular}

D

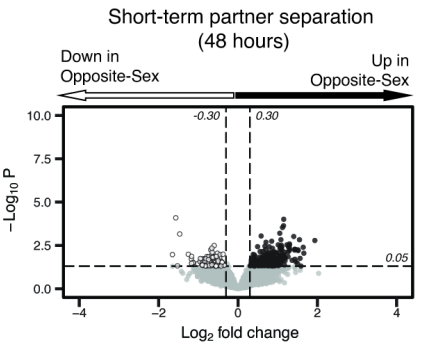

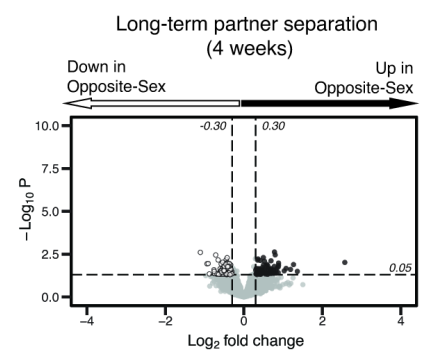

E

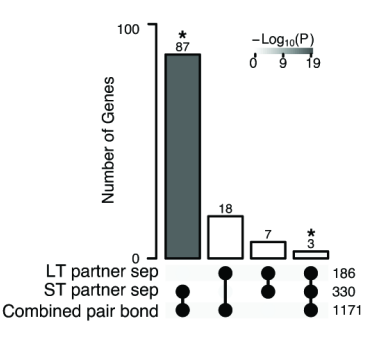

F

Combined Pair Bond ST separation LT separation
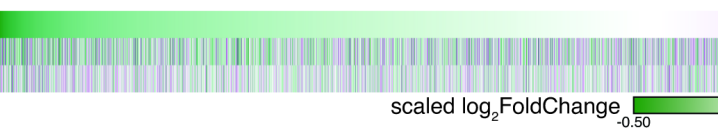

G

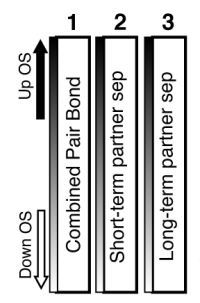

Short-term Separation
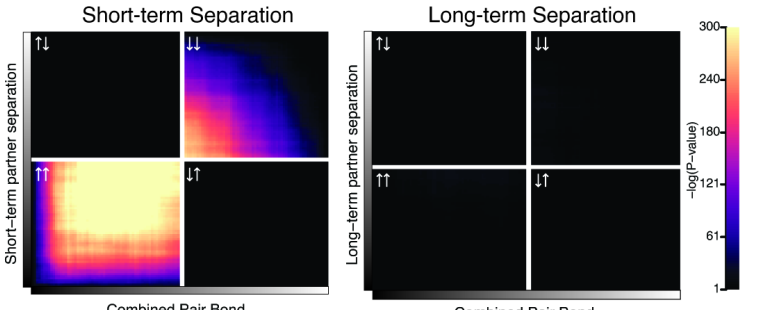

$\mathrm{H}$

Combined Pair Bond

Combined Pair Bond

J

Eroded ST to LT UU genes in DESeq heatmap

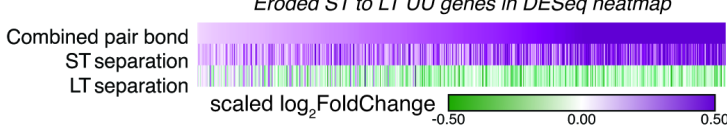

$\mathrm{L}$ Combined pair bond
ST separation
LT separation
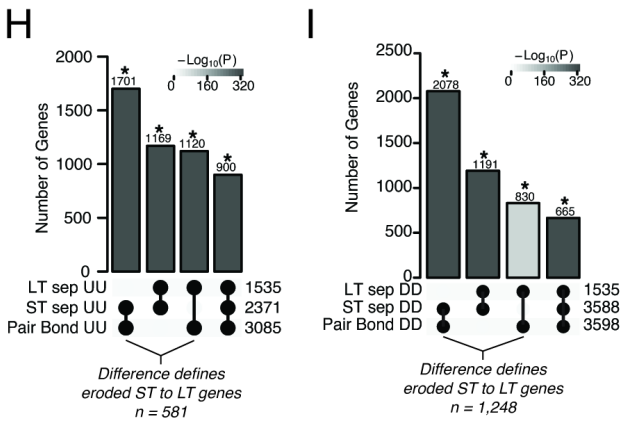

K

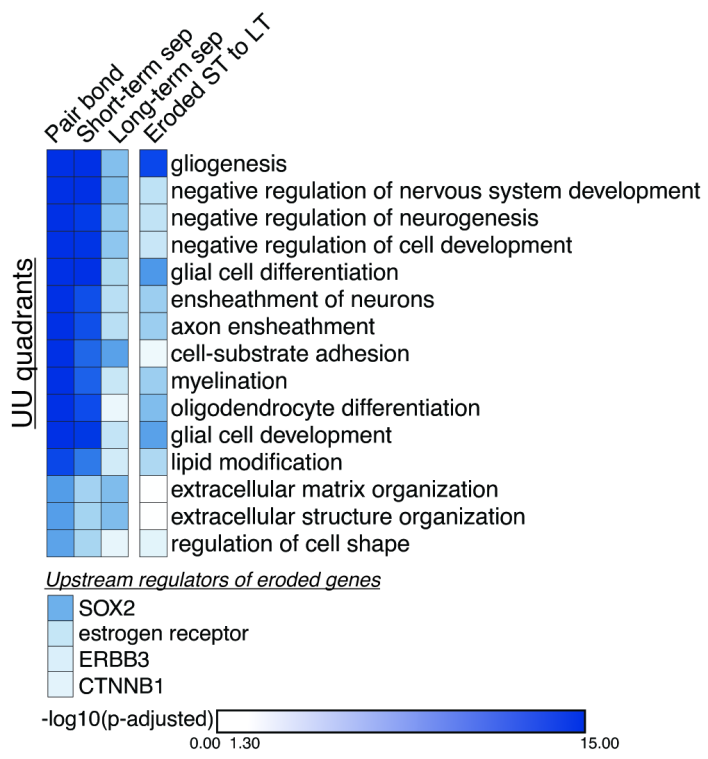

M

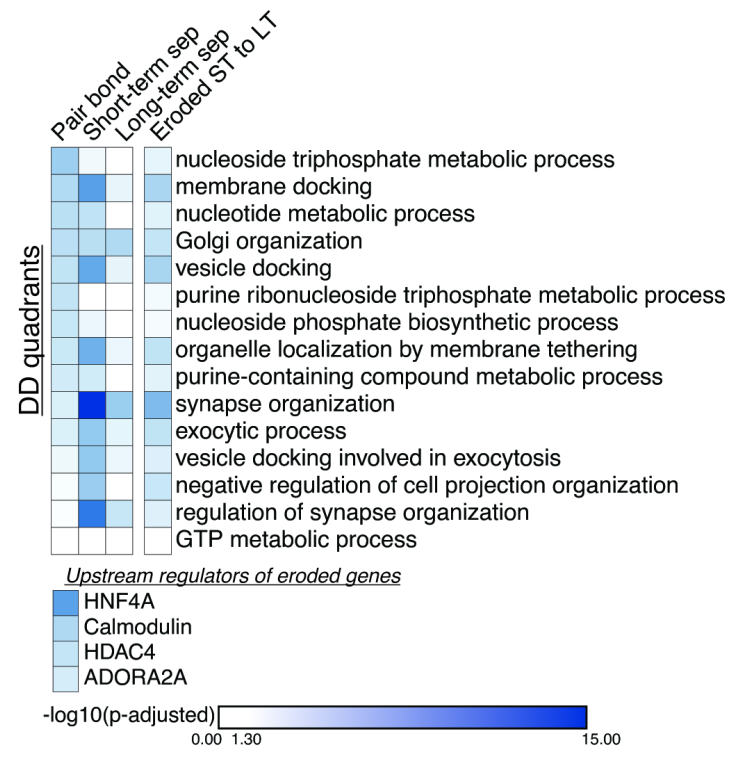

(legend on next page) 
bioRxiv preprint doi: https://doi.org/10.1101/2021.07.14.452355; this version posted July 14, 2021. The copyright holder for this preprint (which was not certified by peer review) is the author/funder, who has granted bioRxiv a license to display the preprint in perpetuity. It is made available under aCC-BY-NC-ND 4.0 International license.

Sadino et al., 13 July 2021 -BioRxiv

\begin{abstract}
Fig. 3 Prolonged separation erodes pair bond transcriptional signatures. (A) Opposite-sex and same-sex pairs were paired for 2 weeks prior to a baseline partner preference test. Pairs were then separated for either 48 hours (short-term) or 4 weeks (long-term) prior to collecting fresh nucleus accumbens tissue for RNAseq. Animals in the Remain Paired group are analyzed in Figure 2 to define the Combined Pair Bond transcriptional signature. (B) Baseline partner preference scores of males included in RNA sequencing for the opposite- and same-sex separation groups (one-tailed t-test relative to 50\%: oppositesex $\mathrm{T}_{11}=11.56, \mathrm{p}=1.71 \mathrm{X} 10-7$; same-sex $\left.\mathrm{T}_{9}=6.07, \mathrm{p}=1.87 \mathrm{X} 10-4\right)$. Black dotted line indicates a 50\% partner preference score and the grey dotted line indicates 66\%. There were no differences in partner preference score between opposite-sex and same-sex separated animals used for RNAseq (two-tailed t-test: $\mathrm{T}_{14.33}=0.33, \mathrm{p}=0.75$ ). (C) Transcriptional analysis workflow similar to Figure 2. (D) Differential gene expression using DESeq2 where genes upregulated in opposite-sex separated males are to the right (black dots) and genes downregulated are to the left (white dots). (E) Upset plot showing the combined up- and downregulated separation DEGs compared to the combined pair bond DEGs. There are more shared transcripts between the pair bond and short-term separated animals than the pair bond and long-term separated animals. Asterisks and shading indicate statistically significant intersections (Fisher's exact test: pair-bond:short-term sep $x^{2}=31.86, p=6.78 \times 10-19$; pair-bond:long-term sep $\times 2=17.96, p=0.53$ ). (F) Heatmap of the scaled log2FoldChange for every gene where short- and long-term separation is compared to the pair bond gene signature. (G) RRHO using the combined pair bond, short-term separated, and long-term separated ranked transcript lists show that short-term separated animals retain a gene expression pattern concordant with pair bond transcription while there is a dramatic erosion of pair bond gene expression following long-term separation. (H, I) Upset plot showing overlap of genes in the UU or DD quadrants of $2 H$ and $3 G$ RRHO was determined using a Fisher's Exact test. Mirroring the results from DESeq, more overlap is found between the pair bond and short-term separated gene lists than with long-term separated gene lists. The difference between the intersections of the pair-bond:short-term sep. and pair-bond:long-term sep. was used to define the eroded gene lists. (J, L) The RRHO eroded gene lists were used to filter the DESeq heatmap in $2 F$. Eroded genes from the UU quadrants show upregulation during pair bonding and short-term separation but downregulation after long-term separation (J) while the eroded genes from the DD quadrants show the opposite pattern (L). (K, M) GO and IPA analysis of RRHO quadrant gene lists and eroded gene lists. Scale represents the - $\log 10$ (p-adjusted) where any non-significant terms are white. (K) Glial associated GO terms of the UU quadrants are less significantly represented after separation and are highly significantly represented among the eroded genes. Significant upstream regulators of the eroded genes include Sox2, estrogen receptor, Erbb3, and Ctnnb1. (M) Vesicle docking and synapse organization associated GO terms of the DD quadrants are less significantly represented after separation and are more significantly represented among the eroded genes. Significant upstream regulators of the eroded genes include Hnf4a, Calmodulin, Hdac4, and Adora2a.
\end{abstract}

and least between the combined pair bond vs long-term separation, a result parsimonious with the most extensive erosion of pair bond transcription occurring after long-term separation (Fig. 3H: all stats in Table S1, genes in Table S2). The same trend was observed for transcripts in the DD quadrants (Fig. 3I, all stats in Table S1, genes in Table S2). We next identified genes whose differential expression in opposite-sex pairs eroded as a function of separation time. We reasoned that such genes are involved in biological pathways that likely regulate adapting to partner loss and prime an animal to form a new pair bond. Pair bond transcripts no longer found between short-term and longterm separation (i.e. eroded transcripts) include $581 \mathrm{UU}$ quadrant transcripts and 1,248 DD quadrant transcripts (Fig. 3H, I). We next filtered the genome-wide expression data of Fig. 3F by the eroded gene lists (Fig. 3J, L). The eroded UU genes are strongly upregulated in a stable pair bond and show dramatic downregulation following longterm separation (Fig. 3J). Thus, the eroded UU genes and their associated biological processes may represent key aspects of adapting to partner loss. There was also a corresponding reduction in DD gene overlap between a stable pair bond and following separation, which identifies transcripts that become upregulated after long-term separation (Fig. 3L).

Finally, we determined how the separation induced transcriptional changes affected the underlying pair-bond associated biological processes. We used the pair-bond associated GO terms from Fig. 2J as a reference point to determine if these terms were still significantly associated with the underlying transcriptional profile following partner separation (Fig. 3K, M). We anticipated that separation would reduce the prevalence of the pairbond associated GO terms and that the eroded genes would be highly enriched for these terms. For transcripts in the UU quadrants, after short-term separation the pair-bond associated GO terms are still significantly enriched to a similar, though lessened, degree. After long-term separation the underlying transcriptional signature is not as a significantly associated with the pair-bond GO terms. Further, the eroded transcripts are associated with glialassociated terms suggesting an important role for glia in forming pair bonds and adapting to partner loss. The role for glia in mediating adapting to partner loss was also seen in the IPA pathway for glioblastoma multiforme signaling and glioma signaling (Fig. S3C). Upstream regulators inducing the erosion of these glial-associated genes include Sox2, estrogen receptor, Erbb3, and Ctnnb1 (Fig. 3K, Fig. S3C). We observe a similar trend in the DD quadrants where the pair-bond associated GO terms become less representative of the underlying transcriptional signature following long-term separation but are associated with the eroded genes, though to a lesser degree (Fig. 3M). IPA pathways activated in a stable pair bond (endocannabinoid signaling, synaptogenesis, and dopamine signaling) are significantly represented among the eroded DD genes (Fig. S3C). Upstream regulators for the eroded DD transcripts 
Sadino et al., 13 July $2021-$ BioRxiv

include Hnf4a, Calmodulin, Hdac4, and Adora2a (Fig. 3M, Fig. S3C).

\section{Neuronal interrogation of long-term partner separation reveals gene clusters associated with pair bond disruption and loss adaptation}

Our tissue-level analysis of gene expression strongly implicated a role for glia in bond formation and loss, and we reasoned that relevant neuronal transcriptional changes may have been masked by these prominent glial transcriptional signals. Thus, we specifically examined separation-induced neuronal transcriptional changes by pioneering translating ribosome affinity purification in voles (vTRAP) $(45,46)$. The vTRAP construct, DIO-eGFPRPL10a, is a double-floxed inverse orientation eGFP-tagged ribosomal subunit that uses the prairie vole RPL10a gene sequence (Fig. 4A; Fig. S4A). The vTRAP construct is paired with Cre-recombinase for inducible, cell-type specific expression. AAV-mediated delivery of hSyn-Cre, which drives neuron-specific Cre-recombinase expression, and hSyn-DIO-vTRAP vectors results in neuronal expression of GFP-tagged ribosomes. Subsequent immunoprecipitation of the tagged ribosomes provides a means to isolate neuronspecific, actively-translating mRNAs $(45,46)$. vTRAP is Credependent as confirmed by bilateral NAc injections of +/Cre (Fig. S5A). We performed vTRAP immunoprecipitation of 3 cohorts: 1) opposite-sex long-term separated, 2) opposite-sex long-term remain paired, and 3) same-sex long-term separated.

We first identified neuronally-enriched transcripts by comparing the transcriptional profiles of the input fraction (equivalent to bulk RNA-seq) and pulldown fraction of the animals from the TRAP groups specified above. We predicted that the pulldown fraction would be enriched for neuronal markers and depleted in glialassociated transcripts. Differential expression analysis revealed that most transcripts are depleted in the pulldown fraction as expected (Fig. 4B). GO terms associated with DEGs enriched in vTRAP pulldowns refer to the mitochondria and cellular respiration while GO terms associated with the depleted DEGs include gliogenesis and myelination, confirming that hSyn-vTRAP successfully isolates neuronally-enriched transcripts and filters out genes associated with gliogenesis (Fig. 4C). We further validated that vTRAP enriches for neuronal transcripts by using weighted correlation network analysis (WGCNA) to identify gene modules significantly correlated with the input (negative correlation) or pulldown (positive correlation) fractions (Fig. 4D, Fig. S5B) (47). Of note, two of the most significantly positively correlated modules, blue and grey60, contain multiple neuronal genes of interest such as Drd1a, Drd2, Oprk1, Fosb, and Pdyn, which have previously been implicated in pair bonding and reward learning $(22,29,48)$.

Together, WGCNA and DESeq analyses indicated that hSyn-driven vTRAP enriches for neuronal transcripts. We used the intersection of these analyses to define 2,365 neuronal-enriched genes within our dataset (Fig. 4E). Enrichr analysis for cell type indicates that the neuronal enriched genes are predominantly associated with the nucleus accumbens and dorsal striatum (Fig. 4E) (49-51). We next queried potential neuronal transcriptional changes in our tissue-level RNAseq data. To ensure that the tissuelevel expression is representative of neuron-specific expression we compared the expression patterns of the neuronal enriched genes in the input and pulldown fractions of opposite- vs same-sex long-term separated males (Fig. S5C). The strong similarity in expression validates that bulk sequencing expression can be used to infer the neuronal expression of those same genes (Fig. S5C). As such, we filtered the tissue-level RNAseq dataset from Fig. 3F by the neuronal enriched gene list of Fig. $4 \mathrm{E}$ to track neuronal gene expression throughout partner loss. We then performed unsupervised clustering of all genes (Fig. 4F, top) and neuronal enriched genes (Fig. 4F, bottom). We identified three neuronal-enriched gene clusters that had particularly interesting expression patterns. Cluster 1 consists of genes that were upregulated in opposite-sex paired males during pair bonding and short-term separation and downregulated following long-term separation. GO terms associated with these genes include adult walking behavior, a term that contains the autismassociated gene Shank3 (Fig. 4G) $(52,53)$. Cluster 2 transcripts are downregulated in pair bonded males and then robustly upregulated in opposite-sex separated males at the short-term and, to a lesser extent, at the long-term timepoint indicating an acute transcriptional response to pair bond disruption. The GO terms associated with these genes suggest an involvement of dopaminergic systems (Drd1a and Drd2) and learning processes potentially engaged to adapt to loss (Fig. 4G). Finally, Cluster 3 contains genes that were downregulated in pair bonded males and became upregulated only after long-term opposite-sex partner separation, representing transcripts that may help prime the vole to form a new bond. The GO 
bioRxiv preprint doi: https://doi.org/10.1101/2021.07.14.452355; this version posted July 14, 2021. The copyright holder for this preprint (which was not certified by peer review) is the author/funder, who has granted bioRxiv a license to display the preprint in perpetuity. It is made available under aCC-BY-NC-ND 4.0 International license.

Sadino et al., 13 July 2021 -BioRxiv

A

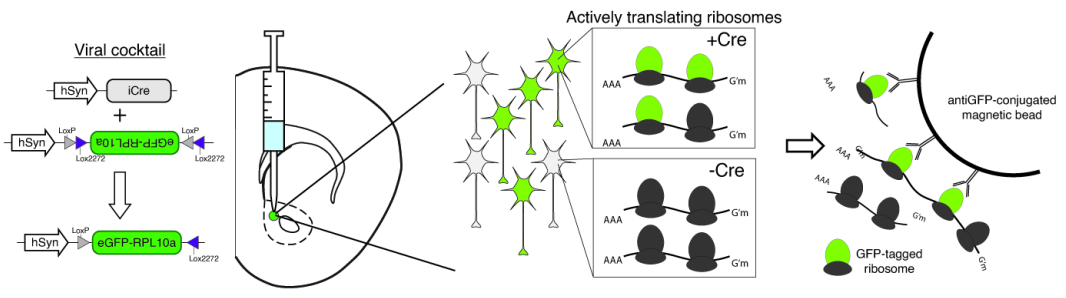

B

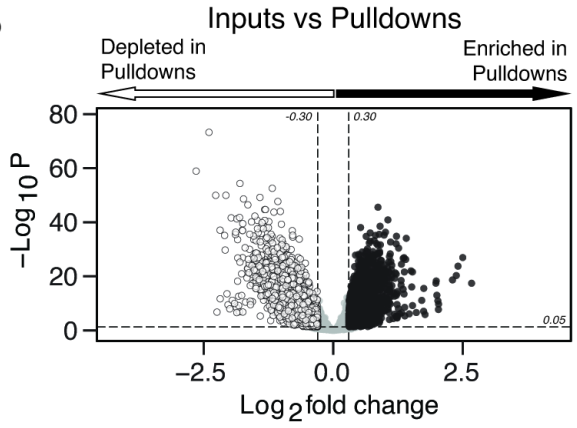

E

\section{Defining neuron enriched genes}

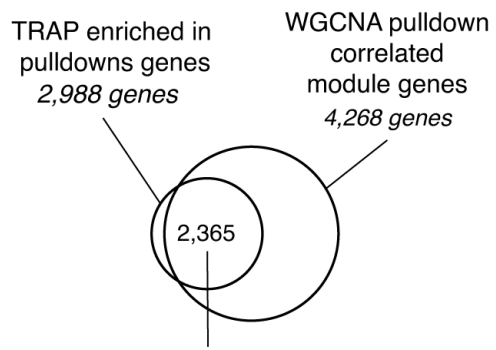

Neuronal enriched genes

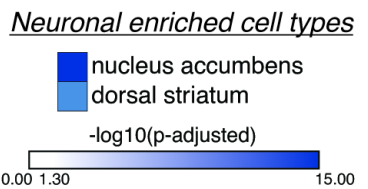
oligodendrocyte differentiation

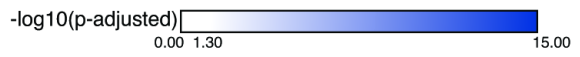

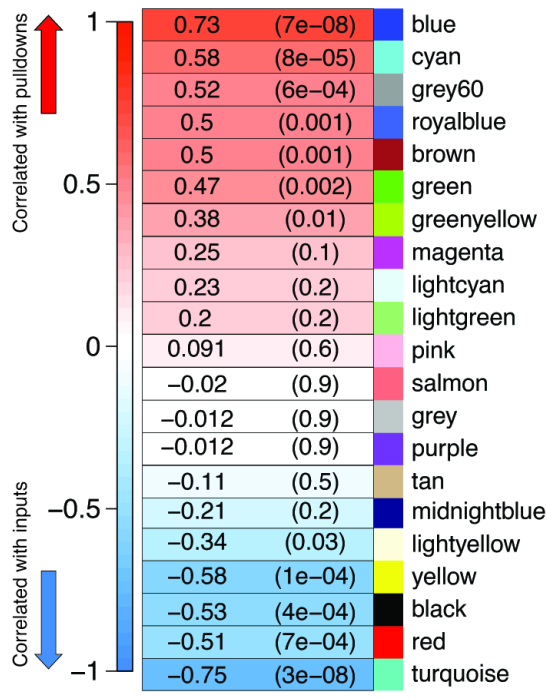

Input vs Pulldown

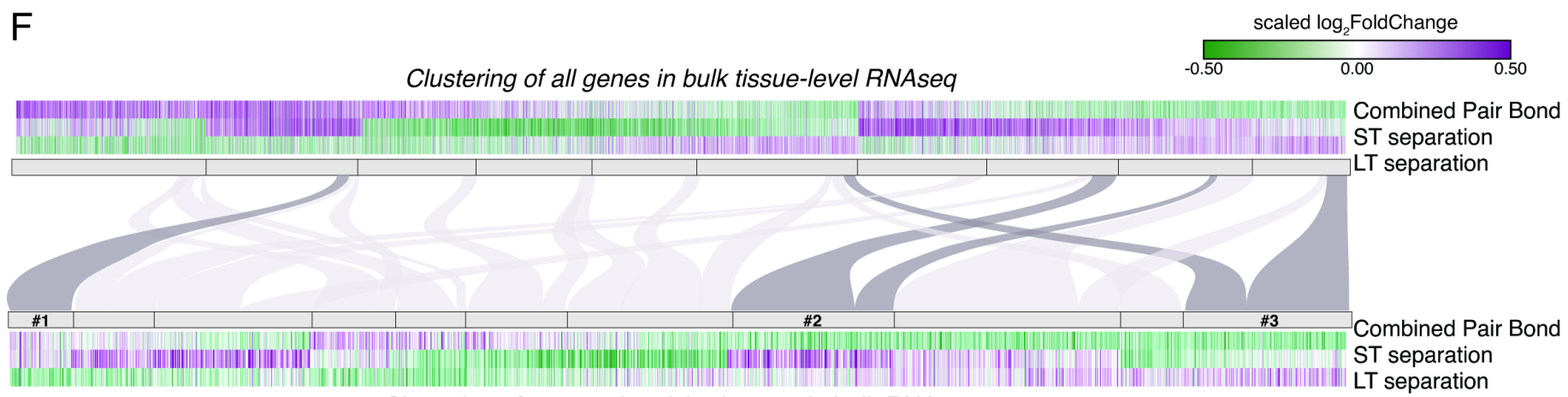

Clustering of neuronal enriched genes in bulk RNAseq

G

Neuronal cluster \#1

ATP metabolic process

purine nucleoside diphosphate metabolic process

adult walking behavior

adult behavior

regulation of organelle transport along microtubule

walking behavior
Neuronal cluster \#2

ncRNA processing

response to amphetamine

response to amine

postsynaptic modulation of chemical synaptic transmission

learning

locomotory behavior

Prominent genes Drd1a, Drd2, Adora2a
- $\log 10$ (p-adjusted)

\section{Neuronal cluster \#3}

protein-containing complex localization mitochondrion organization

NADH dehydrogenase complex assembly mitochondrial respiratory chain complex assembly mRNA processing

ATP metabolic process

$$
\frac{\text { Prominent genes }}{\text { Hras, Slc25a4 }}
$$

Fig. 4 vTRAP elucidates separation-induced transcriptional changes specifically in neurons. (A) Schematic of the vTRAP system. AAV-mediated delivery of hSyn-Cre, which drives neuron-specific Cre-recombinase expression, and DIO-vTRAP vectors results in neuronal expression of GFP-tagged ribosomes. Subsequent immunoprecipitation of the tagged ribosomes isolates neuron-specific, actively-translating mRNAs. (legend continued on next page) 
Sadino et al., 13 July $2021-$ BioRxiv

(B) Transcripts enriched in the immunoprecipitation pulldowns compared to the input fractions. Differential expression skews towards depletion. (C) Gene Ontology analysis using mouse ontology terms indicates that transcripts associated with the mitochondria and cellular respiration are enriched while transcripts associated with gliogenesis and myelination are depleted, supporting neuronal targeting of RPL10-GFP. (D) We further validated the use of vTRAP via WGCNA to identify gene modules correlated with the input (negative correlation) or pulldown (positive correlation) fractions. The grey60 module contains neuronal transcripts of interest such as Drd1a, Fosb, Oprk1, and Pdyn while the blue module contains Drd2. (E) Neuronal enriched genes2,365 genes - were defined as the intersection of the Input v Pulldown enriched DEGs and the WGCNA pulldown-correlated modules (modules grey60, blue, greenyellow, brown, green, royalblue, and cyan). When using Enrichr to search for cell types associated with the neuronal enriched gene list, nucleus accumbens is the most prominent and is highly significant followed by dorsal striatum. Scale represents $-\log _{10}(\mathrm{p}$-adjusted). (F) Hierarchical clustering using one minus Pearson correlation with complete linkage of bulk tissue-level sequencing for all genes (top heatmap) and neuronal enriched genes (bottom heatmap) for the combined pair bond, short-term separation, and long-term separation. The scale indicates scaled $\log _{2}$ FoldChange. The mapping of neuronal clusters to the all genes clusters is visualized via a Sankey plot. Neuronal clusters of interest are highlighted by dark grey links. (G) Gene Ontology analysis using mouse terms for each neuronal cluster of interest. Genes of interest that occur in multiple terms are referred to as prominent genes. Scale represents $-\log _{10}$ (p-adjusted). GO terms associated with neuronal cluster \#1 are not significant due to the small number of genes in that cluster.

terms associated with this cluster are primarily implicated in cellular metabolism (Fig. 4G). IPA revealed that neuronal clusters 2 and 3 were also enriched for estrogen receptor signaling and oxidative phosphorylation pathways with cluster 2 also implicating opioid signaling pathways (Fig. S3D). Steroid and opioid signaling have been extensively implicated in pair bonding $(22,29,37,54-58)$.

\section{Discussion}

Our data provide the first comprehensive assessment of social behavior and accumbal transcription in male prairie voles adapting to partner loss. Prairie voles, unlike laboratory mice and rats, form lifelong pair bonds and affiliative same-sex relationships, making them ideal for studying the neurobiology of bonding and loss $(6,9,14$, 15, 17-19). Within this context, direct comparison of opposite- versus same-sex paired animals serves two crucial purposes. First, it identifies the behavioral and transcriptional responses specific to opposite-sex pair bonding and partner loss as opposed to other affiliative relationships. Second, it provides an important control for the effects of social isolation, which is stressful for all social animals examined to date and results in altered NAc transcription even if the species cannot form pair bonds (59, 60 ). By leveraging this comparison across our experiments, we showed that partner preference and selective aggression in opposite-sex-separated males mirrors that of same-sex paired males and persists following 4 weeks of separation despite a large erosion in pair bond-associated transcription. This suggests that substantial changes in accumbal gene expression may contribute to priming the vole to form a new bond but are not sufficient to erase an existing bond. In addition, we demonstrate the utility of vTRAP for interrogating neuron-specific responses to bonding and separation that are obscured when analyzing bulk sequencing results. Together, these results expand the utility of monogamous prairie voles for future behavioral and molecular-genetic studies of partner loss and loss adaptation.

\section{Partner preference persists despite extended separation}

We observed overall similarity and persistence in social behaviors exhibited by opposite-sex and same-sex paired prairie voles, both of which displayed a partner preference despite prolonged separation. The persistence of partner preference following 4 weeks of separation is particularly notable in the context of rodent social memory. The majority of laboratory rodents form fleeting social memories that are not detectable after a few days to a week (61-64). Displaying a partner preference requires that an animal remembers their partner. Our results indicate that, for both an opposite-sex and same-sex partner, prairie voles are capable of much longer-lasting social memories than other laboratory rodents.

Our results also contradict prior work demonstrating dissolution of partner preference in male voles after four weeks of separation (65), although this may be due to differences in study design. Potential explanations include differences in repeated measures design (within animal vs separate cohorts), differences in the underlying genetics between prairie vole colonies, differences in pregnancy controls (tubal ligation vs ovariectomy and hormone replacement), and pairing duration prior to separation (2 weeks vs 24 hours). Regardless, our results suggest that male voles have the capacity to remember and potentially resume an affiliative relationship despite prolonged separation and isolation.

\section{Pair bonding results in consistent glia-associated transcriptional changes that erode following prolonged separation}

We first asked whether bonding-induced gene expression is stable as long as pairs remain cohoused (22, $23,29,30)$. Using three orthogonal analytical methods, we 
found remarkable consistency in the transcriptional pattern of opposite- vs same-sex pairs co-housed for 2 or 6 weeks. Prior work on social dominance suggests that such stable transcriptional changes are critical to maintain different behavioral states (26-28). However, to our knowledge, this is the first demonstration that a similar conceptual underpinning may maintain mature pair bonds.

Consistently upregulated transcripts in pair bonded males are associated with glia and extracellular matrix organization while downregulated transcripts are associated with nucleoside processes, synaptic plasticity, and vesicle trafficking. Together, these terms implicate processes central to neuronal remodeling and synaptic plasticity. Of note, while our analyses do identify a number of mitochondrial GO terms, these terms are not as strongly implicated in our analyses as in previous reports (20). However, mitochondrial terms are still represented and, together, our studies support that mitochondrial dynamics in the NAc are likely important for male prairie vole pair bonding. This is consistent with a model in which pair bonding and separation induce neuronal plasticity and synapse re-organization, which require large amounts of energy and induce mitochondrial redistribution within neurons $(66,67)$.

We next found that pair bond transcriptional signatures erode as a function of separation duration. In particular, the notable reduction of DEGs following longterm separation suggests that the transcriptional signatures of opposite- versus same-sex separated animals largely converge after extended separation. Likewise, via RRHO, we found that there is a considerable reduction in concordant pair bond gene expression following long-term, but not short-term, separation. When we asked which transcripts and associated biological processes erode over this timeframe, we found that they largely corresponded with those evident in the stable pair bond transcriptional signature. Specifically, transcripts associated with glia, extracellular matrix organization, synapse organization, and vesicle docking are stably expressed in pair bonded males but eroded following long-term separation. The erosion of the pair bond transcriptional signature over time in opposite-sex separated males may be central to adapting to partner loss in order to form a new pair bond if given the opportunity.

Our analyses strongly indicate a role of glia in regulating pair bonding and the response to partner separation. Transcripts associated with gliogenesis, glia cell differentiation, and myelination are upregulated in stable pair bonds, potentially reflecting the importance of glial cells in sculpting and refining neural circuits in response to social experience $(37,68,69)$. Similar GO terms - with the addition of oligodendrocyte differentiation - are associated with eroded pair bond transcripts after long-term partner separation. Demyelination and oligodendrocyte dysfunction are seen in mice and rats after chronic social stress and in individuals with depression (70-72). One mechanism for social deprivation-mediated demyelination and impaired oligodendrocyte maturation is by reduced flux through Erbb3-based signaling pathways $(73,74)$. Of note, Erbb3 is one of 24 genes whose differential expression is conserved in taxonomically diverse monogamous species when compared to closely-related non-monogamous relatives (75). We find Erbb3 upregulated during pair bonding through short-term separation but it is no longer upregulated following long-term separation. Further, IPA analysis indicates that Erbb3 is an upstream regulator of the eroded genes. Thus, it is possible that long-term separation is downregulating Erbb3-associated pathways resulting in reduced myelination and disrupting oligodendrocytes specifically in opposite-sex-separated animals. In adult mice, social reintroduction rescued abnormal myelination phenotypes suggesting a role for oligodendrocyte plasticity in social behavior (74). An important future direction is whether similar plasticity occurs in voles; does bonding with a new partner re-engage these glial processes?

Finally, we reasoned that the prominence of glialrelated terms may have masked important neuronal transcriptional changes. To hone in on neuronal transcriptional changes specifically, we developed translating ribosome affinity purification in prairie voles (vTRAP). We used DESeq and WGCNA to identify neuronal enriched genes, which show a $1.2-8.8$ fold enrichment in neuronal pulldowns compared to the tissuelevel sequencing data. We then queried expression changes specifically among these genes as a function of pair bonding and partner separation. With this enhanced sensitivity, we were able to identify three neuronal gene expression patterns that are differentially sensitive to partner loss. Notably, dopamine associated genes are robustly upregulated in neurons after acute separation, possibly reflecting a withdrawal-like state (76-78). Finally, genes associated with anxiety and energy production are upregulated only after long-term separation, possibly to facilitate finding a new partner. Together with our tissue level sequencing implicating the role of glia, vTRAP provides a more nuanced understanding of how the pair 
Sadino et al., 13 July $2021-$ BioRxiv

bond neuronal transcriptional signature responds to partner separation over time.

\section{Limitations and future directions}

A notable aspect of our results is that the substantial erosion of pair-bond transcription in the NAc after long-term separation is not matched by a similar magnitude change in behavior, despite evidence that changes in gene expression in this brain region help maintain bonds over time $(23,30,79)$. One potential explanation is that our study only examined responses in the NAc, and transcriptional changes in other brain regions may also be crucial for forming and maintaining a pair bond and responding to partner separation $(21,24,42,80)$. Another possible explanation is that, while the pair bond signature is substantially eroded, it is not completely erased. Therefore, there could be a subset of transcripts that retains pair-bond-induced expression even after prolonged separation. Additionally, RNAseq cannot detect changes in proteins with low turnover rates. The residual pair bond transcriptional signature, and/or long-lasting proteomic changes, may be sufficient to maintain partner preference and selective aggression in opposite-sex-separated males. Alternatively, transcriptional changes in a small subset of cells, which may be undetectable in tissue-level RNAseq, may contribute to the persistence of partner preference. To address the latter, we began to narrow our inquiry through the development of vTRAP to isolate actively translating mRNAs from specific cell types-in this case neurons. Recent advances in cell-type and projection-specific targeting via Cre recombinase and cell-type specific promoters deployable via AAV will further broaden application of vTRAP in voles $(81,82)$. Additionally, refined approaches, such as single-cell RNAseq, have the possibility of greatly enhancing our understanding of the specific cell populations regulating complex behaviors.

Finally, it is worth noting that the persistence of partner preference in our paradigm may have been facilitated by the study design. Unlike in the wild where there are potential opportunities to rebond upon partner loss, the separated males in this study had no opportunity to form a new bond. Under such conditions, it may be advantageous to maintain behaviors that would revive the bond if a partner returned. A more ethologically relevant question may be how long it takes before a separated vole is capable of forming a new bond. Previous work from our lab has shown that male voles can form a new, stable pair bond that supplants the original bond after four weeks of separation but not sooner (16). This would suggest that although partner preference remained intact in the current study, these males were likely capable of forming a new bond if the opportunity arose, a capacity that may arise from the erosion of pair bond transcription. Such an explanation is parsimonious with the human experience. We do not forget our previous bonds; rather we integrate the loss in order to continue on with life.

In sum, we provide foundational assessments that are integral for establishing prairie voles as a relevant model for studying loss. Here we describe that, in the absence of new bonding opportunities, the original pair bond will persist for at least 4 weeks post-partnerseparation. However, prior work indicates that there is still an adaptive process occurring within this time frame that primes a vole to form a new bond if available (16). Our work suggests that this adaptation is mediated at least in part by an erosion of bond-related transcription in reward-related brain regions that largely precedes bond dissolution at the behavioral level. Future studies are needed to ask whether repression of key pair bond transcriptional changes is sufficient to facilitate re-bonding with a new partner. Together, this work and future studies may help shed light on the pain associated with loss, as well as how we integrate grief in order to meaningfully reengage with life.

\section{Materials and Methods}

\section{Animals}

Sexually naive adult prairie voles (Microtus ochrogaster, $\mathrm{N}=$ 232: 172M, 60F, P60 - P168 at experiment start) were bred in-house in a colony originating from a cross between voles obtained from colonies at Emory University and University of California Davis, both of which were established from wild animals collected in Illinois. Animals were weaned at 21 days and housed in same-sex groups of $2-4$ animals in standard static rodent cages $(7.5 \times 11.75 \times 5$ in. $)$ with ad-lib water, rabbit chow (5326-3 by PMI Lab Diet) supplemented with alfalfa cubes, sunflower seeds, cotton nestlets, and igloos for enrichment until initiation of the experiment. All voles were aged between post-natal day 60 and $\sim 180$ at the start of the experiment. Throughout the experiment, animals were housed in smaller static rodent cages (11.0 in. x 8.0 in. x 6.5 in.) with ad-lib water, rabbit chow (5326-3 by PMI Lab Diet), and cotton nestlets. They were kept at 23$26^{\circ} \mathrm{C}$ with a $10: 14$ dark:light cycle to facilitate breeding. All 
procedures were performed in accordance with standard ethical guidelines (National Institutes of Health Guide for the Care and Use of Laboratory Animals) and approved by the Institutional Animal Care and Use Committee at the University of Colorado Boulder.

\section{Tubal ligations}

All females in opposite-sex pairs were tubally ligated to avoid confounds of pregnancy, but to keep the ovaries intact as to not impact hormonal function (Souza et al., 2019). Females were anaesthetized with a mixture of $2 \%$ Isoflurane and $\mathrm{O}_{2}$ gas and depth of anesthetize was monitored by toe pinch throughout surgery. Prior to surgery, animals were weighed and given subcutaneous injections of the analgesic Meloxicam SR $(4.0 \mathrm{mg} / \mathrm{kg}$, Zoopharm) and saline (1 mL, Nurse Assist). Each fallopian tube was cauterized and bisected through lumbar inscitions on each side of the body using standard sterile practices. Animals were monitored once a day, for three days and staples were removed one week post-surgery. Females were given 2 weeks to recover before pairing with a male.

\section{Pairing}

For the RNAseq portion of the experiment, opposite-sex, non-sibling pairs ranged in age between P98 and P168 (average $\sim$ P122) and same-sex, sibling pairs ranged in age between P60 to P163 (average P111) at pairing. For the behavioral experiments, opposite-sex, non-sibling pairs ranged in age between P60 and P141 (average P85) and same-sex, sibling pairs range in age between P62 and P79 (average $\sim$ P76) at pairing. For RNAseq and behavior testing, at pairing, pairs were transferred into smaller static rodent cages (11.0 in. $x 8.0$ in. x 6.5 in.) with ad libidum water and rabbit chow (5326-3 by PMI Lab Diet), a cotton nestlet, and an igloo. Females were not induced. Partners cohabitated undisturbed, except for weekly cage changing performed by the experimenter, for 2 weeks prior to the baseline partner preference test (PPT). For same-sex pairings, sibling pairs from the same homecage were moved to smaller static rodent cages in the same way as the opposite-sex pairs. After the baseline PPT, behavior experiment animals were separated into individual cages for the remainder of the experimental timeline. In contrast, after the baseline PPT, RNAseq experimental animals were either returned to their partner (Remain Paired condition) or were separated into individual cages (Separated condition).

\section{Separation for behavioral tests}

Immediately following the baseline PPT, each pair was separated into fresh small static rodent cages (11.0 in. x 8.0 in. $x 6.5$ in.) with ad libidum water and rabbit chow (5326-3 by PMI Lab Diet), a cotton nestlet, and an igloo. Animals were placed approximately a foot away from each other to eliminate visual cues of their partner. Each animal in the pair remained in their individual cages undisturbed for either 48 hours (short-term separation) or 4 weeks (shortterm separation) except for weekly cage changes done by the experimenter. At each separation time point, experimental animals performed a partner preference test (PPT) and resident intruder test (RIT) on successive days approximately 24 hours apart starting between 9:00 and 10:00 AM each day. Animals were returned back to their original home cages between testing days and transferred to fresh cages $24 \mathrm{hrs}$ after RIT for each time point. Cage changes were done at identical intervals for each cohort prior to behavioral testing.

\section{Partner Preference Test (PPT)}

PPT was carried out as described by TH Ahern to assess selective partner affiliation prior to partner separation and were performed between the hours of 9:00 and 13:00 each testing day (83). Each 3 hour test was recorded by overhead cameras (Panasonic WVCP304) that film two boxes simultaneously. The test animal is placed in the middle chamber for 10 minutes with the cage dividers still in place. The test begins when the cage dividers are removed and the test animal is allowed free range of all three chambers for the duration of the test. The movement of the test animal was tracked post-hoc using Topscan High-Throughput software (v3.0, Cleversys Inc.) and frame by frame behavioral data was analyzed using a custom Python script (https://github.com/donaldsonlabdonald) to calculate the average distance between the test animal and tethered animals when in the same chamber, time spent huddling with each tethered animal, and total distance traveled. This data was then used to generate a number of behavior metrics including the partner preference score (Partner Huddle/Partner + Novel Huddle) (https://Fig.share.com/authors/zoe_donaldson_colorado_e du_Donaldson/6883910).

\section{Resident Intruder Test (RIT)}

Resident males were tested in their home cages (11.0 in. $x$ 8.0 in. $x 6.5$ in.). Food hoppers and igloos were removed 
Sadino et al., 13 July 2021-BioRxiv

prior to the start of the test to allow video recording from the side using Sony Handycams (DCR-SX85). Intruder males were briefly anaesthetized with a mixture of $\sim 2 \%$ Isoflurane to shave their backs for identification during behavior scoring and allowed to fully recover in an empty cage. Intruders were then placed in the resident's home cage and their behavior was filmed for 10 minutes. After the test, the intruders were returned to their home cages and the residents were returned their food hopper and igloos in their original home cage. Behavior scoring was done in BORIS (v7.10.2) by the same blind observer for all RIT tests. Behaviors scored included tumble fighting (aggression), chasing (aggression), defensive postures (aggression), anogenital sniffing (investigation), social investigation (investigation), huddling (affiliation), autogrooming (nonsocial), and digging (non-social). Definitions of scored behaviors can be found in Table S1. Behavior scores were then analyzed in $\mathrm{R}$ (v4.0.4).

\section{Statistical analysis for behavioral experiments}

All statistical analyses were performed in R (v4.0.4) using the base t.test package, base aov package, and the survival package (v3.2-7). Visualization was done using ggplot2 (v3.3.3), ggpubr (v0.4.0), and Adobe Illustrator (25.2.3). All statistical results can be found in Table S1. For PPT, we first determined if partner preference significantly changed from $50 \%$ at each time point (baseline, ST, or LT) and for each pairing type (OS or SS) by using an unpaired, onesided Student's t-test against a null value of 50. We also performed a 2-way repeated measures ANOVA with Tukey's post-hoc test to determine main effects of, and interactions between, the pairing type and separation duration. Next, we calculated partner preference change scores for all conditions and compared the variance between pairing types using a F-test of variance. Finally, we determined changes to partner and novel huddling latency using the built in log-rank test of the survival package. For RIT, we determined any significant changes to the duration or bouts of all scored behaviors and behavior categories using a 2-way repeated measures ANOVA with Tukey's post-hoc test. Additionally, to determine the latency to resident tumble fighting we used the built in logRank test of the survminer package (v0.4.8).

\section{Generation of vole optimized hSyn-DIO-eGFP-RPL10a construct for vTRAP}

Prior to generating the vTRAP viral construct, the nucleotide and amino acid sequences for RPL10a in mice and prairie voles were compared. To assess nucleotide homology, the mouse RPL10a cDNA sequence was aligned to the vole RPL10a cDNA sequence using BLASTN (v2.8.033) with a percent identity of $93 \%$ and an E-value of 0.0 (Fig. S3A). The mouse RPL10a cDNA sequence was input into BLASTX (v2.8.033), and the resulting protein alignment against the prairie vole RPL10a amino acid sequence was $100 \%$ identical with an E-value of $3 \mathrm{E}^{-145}$ (Fig. S3B). Although the amino acid sequences are identical between mice and prairie voles, we generated the VTRAP construct using the prairie vole RPL10a cDNA sequence to account for possible species-specific differences in aminoacyl-tTRNA concentrations.

To generate the TRAP construct, the DNA fragment containing the vole RPL10a sequence was inserted into the pAAV-hSyn-DIO-eGFP (Addgene plasmid \#50457) backbone. The RPL10a fragment was designed for cloning using the NEBuilder HiFi Assembly method and consisted of a 5' homology arm sequence, a linker sequencecoding for SGRTQISSSSFEF, the vole RPL10a sequence (GenBank XM_005360358.1), and a 3' homology arm sequence (Supplementary Fig.. 1). pAAV-hSyn-DIO-eGFP was digested using restriction endonucleases Asc1 (NEB) and BsrG1-HF (NEB), and the linearized plasmid was purified using the Zymogen Gel DNA Recovery Kit. The cloned plasmid, hereafter referred to as pAAV-hSyn-DIOeGFPpvRPL10a (vTRAP), was generated using theNEBuilder HiFi DNA Assembly Cloning kit and subsequently transfected into 5-alpha F'IqCompetentE. coli(NEB). Transfected bacteria were selected for using carbenicillin $(100 \mathrm{ug} / \mathrm{ml})$ and harvested using the EZDNA Plasmid Mini Kit (Omega) or HiSpeed Plasmid Maxi Kit (Qiagen). The sequence of vTRAP was verified using Sanger sequencing (QuintaraBio) and restriction digest before being sent for viral packaging in an AAV1 serotype (Stanford Neuroscience Gene Vector and Virus Core).

\section{Viral injections}

For stereotaxic surgery, adult prairie voles were anesthetized using isoflurane (3\% induction, $1-2.5 \%$ maintenance) and depth of anesthesia was monitored by breathing and toe pinch reflex. After induction, animals were placed on a heating pad and given an analgesic (2 
$\mathrm{mg} / \mathrm{kg}$ Meloxicam SR, subcutaneous, Zoopharm) and sterile saline ( $1 \mathrm{~mL}$, subcutaneous). Standard sterile surgical procedures were followed to expose and drill through the skull. Nucleus accumbens injection coordinates were adapted from the Allen Mouse Brain Atlas and validated prior to the experiment (AP: -1.7, ML: \pm 1.0, DV: $-4.7,-4.6,-$ $4.5,-4.4,-4.3)$. For each DV coordinate, $100 \mathrm{~nL}$ of virus was dispensed at $0.1 \mathrm{~nL} / \mathrm{sec}$ for a total of $500 \mathrm{~nL}$ using a Nanoject II (Drummond Scientific). After the last injection, the needle remained in place for 10 minutes to allow for diffusion. The glass needle was then removed and the skin was sutured closed (vicryl sutures, eSutures), coasted with lidocaine and antibacterial ointment, and animals recovered in a cage on a heating pad with continued monitoring. The animal's health was monitored for 3 days following surgery and they were allowed to recover in their homecage for 2 weeks prior to the experiment start.

\section{Verification of TRAP Expression}

Cre-dependent expression of the vole optimized eGFPRPL10a custom virus was validated in adult male prairie voles $(\mathrm{n}=1)$ using a within animal design. The left NAc was injected with a viral cocktail of AAV1-DIO-eGFP-RPL10a (2 $\mathrm{uL} ; 5.55 \mathrm{E} 11 \mathrm{vg} / \mathrm{mL}$ ) and AAV1-hSyn-iCre (2 uL; 3.9E12 $\mathrm{vg} / \mathrm{mL}$ ) while the right NAc only received the AAV1-DIOeGFP-RPL10a virus (600 nL; 2.22E+12 vg/mL). Following 2 weeks of recovery to allow for robust viral expression the animal was perfused. The animal received a lethal injection of $0.2 \mathrm{~mL} \mathrm{1:2} \mathrm{ketamine/xylazine} \mathrm{and} \mathrm{was} \mathrm{perfused} \mathrm{with} 1 \mathrm{X}$ PBS followed by $4 \%$ paraformaldehyde (in 1X PBS, Electron Microscopy Sciences) and kept in 4\% PFA overnight. The brain was then placed in $5 \mathrm{~mL}$ of 30\% sucrose for 3 days prior to sectioning on a microtome (Leica JungSM2000R, 50 microns/slice). Sections were wet mounted onto Superfrost Plus glass slides (Thermo Fisher Scientific), cover slipped (ProLong Gold; Invitrogen), and allowed to dry overnight prior to imaging. Prepared slides were then sealed with nailpolish (Electron Microscopy Sciences) and imaged using a Yokogawa CV1000 Confocal Scanner System (University of Colorado Light Microscopy Core Facility). Stitched images were produced using ImageJ (v1.51).

\section{Separation for RNAseq experiments}

Immediately following the baseline PPT, each pair was moved to fresh small static rodent cages (11.0 in. x 8.0 in. $x$ 6.5 in.) with ad libidum water and rabbit chow (5326-3 by PMI Lab Diet), a cotton nestlet, and an igloo. Remain Paired animals were kept with their partner while Separated animals were separated from their partner and moved to a fresh cage while their partner was immediately SAC'd to remove confounds of visual or olfactory cues. All animals remained in their individual cages undisturbed for either 48 hours (acute separation) or 4 weeks (chronic separation) except for weekly cage changes done by the experimenter. After the appropriate separation time point, experimental animals were removed directly from their home cage for rapid decapitation and tissue harvesting.

\section{RNA preparation and sequencing}

Tissue lysis and RNA isolation was adapted from Heiman and performed under RNAse free conditions (45). On each tissue collection day, 2 animals from each experimental cohort were processed, with the exception of same-sex 4 week paired animals who were all collected on the same day. Animals were removed from home cages, rapid decapped, and both hemispheres of the Nucleus Accumbens were hand dissected using sterile, RNAse free razor blades and pooled. During dissection, tissue was kept wet with dissection buffer (HEPES KOH, pH 7.320 mM, 1X HBSS, Glucose $35 \mathrm{mM}$, NaHCO3 $4 \mathrm{mM}$, CHX $10 \mathrm{uL}$ of 1000X stock, DEPC-treated water up to $10 \mathrm{~mL}$ ). Tissue was added to $1 \mathrm{~mL}$ of ice-cold lysis buffer (DEPC-treated water, HEPES $20 \mathrm{mM}, \mathrm{KCl} 150 \mathrm{mM}, \mathrm{MgCl} 2(10 \mathrm{mM})$, DTT $5 \mathrm{uL}$ of 1M stock, CHX $10 \mathrm{uL}$ of 1000X stock, 1 protease inhibitor, RNasin 400 Units, and Superasin 800 Units), immediately homogenized using a Scilogex homogenizer, and kept on wet ice while the remaining animals for the day were processed. The homogenizer tip was thoroughly cleaned in between animals with DEPC-treated water and $70 \%$ ethanol to eliminate cross contamination between samples. Homogenized samples were centrifuged at $4^{\circ} \mathrm{C}$, for 10 minutes at $\sim 3000 \mathrm{Xg}$ and supernatent was transferred to new, pre-chilled tubes. 10\% NP-40 (AG Scientific: \#P-1505) and $300 \mathrm{mM}$ DHPC (Avanti Polar Lipids, Inc.: 850306P-200 $\mathrm{mg}$ ) were added to the supernatent and incubated on ice $\left(4^{\circ} \mathrm{C}\right)$ for 5 minutes. Samples were then centrifuged at $4^{\circ} \mathrm{C}$ for 10 minutes at $16000 \mathrm{Xg}$ and supernatent was transferred to new, pre-chilled tubes. RNA was then extracted and cleaned from the lysate supernatent using the Norgen Total RNA micro kit (cat. \#) according to the manufacturer's instructions. RNA integrity was measured using an Agilent Tapestation prior to library preparation. RINs for all samples were $>7.3$ (average 8.8 ). 
Sadino et al., 13 July $2021-$ BioRxiv

For TRAP samples, dissections and Input fraction RNA were prepared as above. To perform immunoprecipitation pulldowns, affinity matrices were prepared using $300 \mathrm{uL}$ of $10 \mathrm{mg} / \mathrm{mL}$ Streptavidin MyOne T1 Dynabeads (Invitrogen), $150 \mathrm{uL}$ of $1 \mathrm{ug} / \mathrm{uL}$ Biotinylated Protein L (Fisher Scientific), and 50 ug each of eGFP antibodies Htz-19C8 and Htz-19F7 (Memorial-Sloan Kettering Monoclonal Antibody Facility) 2 hours prior to adding Input RNA sample. A small sample of Input RNA was saved for next day clean up ( 250 $\mathrm{uL} /$ sample) and frozen at $-80 \mathrm{C}$ overnight. The remaining Input RNA $(\sim 1 \mathrm{~mL})$ was added to the GFP-conjugated beads and rotated overnight (16-18 hours) at $4{ }^{\circ} \mathrm{C}$. The next day, the flowthrough was saved and stored at $-80 \mathrm{C}$ and bound mRNA was released from the beads using a series of high salt buffer washes (4 washes; 20mM HEPES, $150 \mathrm{mM}$ $\mathrm{KCl}, 10 \mathrm{mM} \mathrm{MgCl} 2,10 \% \mathrm{NP}-40$, RNase-free water, protease inhibitor tablets, $0.5 \mathrm{mM}$ DTT, $100 \mathrm{ug} / \mathrm{ul}$ cycloheximide). The beads were then incubated with the Norgen Total RNA micro kit SKP buffer (300 uL) for 10 minutes at room temperature. The Pulldown supernatant was then saved and processed as above using the kit manufacturer's instructions.

Library preparation for sequencing was done using the KAPA mRNA HyperPrep kit with polyA enrichment according to the manufacturer's instructions for a 100-200 bp insert size and using $1.5 \mathrm{uM}$ TruSeq3-SE adapters. 71 samples were pooled and 3 runs of NextSeq V2 high output 75 cycle (1X75) sequencing produced a total read depth of $\sim 20 \mathrm{M}$ reads/sample. The Illumina sequencing was performed at the BioFrontiers Institute Next-Gen Sequencing Core Facility of University of Colorado Boulder. RNA quantity, RIN, and RNA amount used for library preparation can be found in Table S1.

\section{Sequence mapping and counting}

For quality control, FastQC (0.11.8) and Trimmomatic-Src (v0.38) were used to analyze read quality and to trim the TruSeq3-SE adapters from all samples (84). Additionally, each sequencing run was correlated to each other to ensure that there was not a significant difference in run composition. Reads were aligned to the prairie vole genome (Microtus_ochrogaster.MicOch1.0.dna.toplevel, released Feb 2017; accession GCA_000317375) using HiSat2 (2.1.0) with default options and individual runs for each animal were merged into a single sample using SAMtools (1.9) (85, 86). Reads were then counted using HTseq (0.11.2) with default options using the annotated prairie vole genome
(Microtus_ochrogaster.MicOch1.0.95.gtf, released Feb 2017; accession GCA_000317375) (87). Genes with less than 10 read counts were filtered from downstream analysis and, where necessary, were normalized using transcripts per million (TPM). Where possible, all Ensemble IDs were converted to prairie vole gene IDs using BioMart. If no gene ID was found, the original Ensemble ID was retained. Only genes with greater than 10 counts were included in subsequent analyses (total genes $=21152$, genes $>10$ counts $=12128)$.

\section{Data analysis and visualization}

All sequencing analysis was performed using $\mathrm{R}$ (v4.0.4) (88). The packages used and their specific parameters are described in the relevant sections below. Plots were made using base plot, ggplot2 (v3.3.3), EnhancedVolcano (1.8.0), RRHO2 (v1.0), clusterProfiler (v3.18.1), and Adobe Illustrator (25.2.3) $(35,36,89,90)$.

\section{DESeq}

Differential gene expression was analyzed with the $\mathrm{R}$ package DESeq2 (v1.30.1) following the standard workflow (31). To determine differential gene expression between cohorts a DESeq dataset of all animals was made with the design $\sim$ ParentCode + AgeAtPairing + Cohort to correct for the effect of parentage and age at initial pairing. Other batch effects (processing day, processing order, RIN, etc.) did not have sufficient influence on the model to include. Each result was a pair-wise contrast between the appropriate cohorts with the opposite-sex condition as the experimental and the same-sex condition as the reference. Differential gene expression was defined as $>0.30$ and $<-0.30$ log2FoldChange and p-value $<0.05$. This Log2FoldChange threshold was used for two reasons. 1) A threshold of +/0.20 can be successfully validated by qPCR (32) but 2) we chose a slightly higher threshold as it allows for smaller gene lists that appear more biologically relevant by Gene Ontology analysis. Differential gene expression was visualized using EnhancedVolcano. To determine if there was statistically significant overlap between differentially expressed gene lists compared to all genes $(n=12,128)$ we performed a Fisher's exact test using the SuperExactTest (v1.0.7) package (33). Additionally, we used Morpheus (https://software.broadinstitute.org/morpheus) heatmaps to compare the expression profiles of each DESeq comparison using scaled log2FoldChange values. For each heatmap, a reference dataset ordered the remaining 
datasets such that each column is the same gene and the color represents the scaled $\log _{2}$ FoldChange in each DESeq comparison. In Fig. $2 F$ the short-term pair bond was set as the reference and in Fig. 3F, J, and $L$ the combined pair bond was set as the reference. Gene ontology and pathway analysis of relevant gene lists were performed using the $\mathrm{R}$ package enrichGO with Mus musculus ontology terms (36). Representative terms with an adjusted p-value of $<0.05$ were hand selected. Results were visualized using base $R$ plots modified in Illustrator. All DESeq result data frames can be found in Table S2.

\section{Rank-Rank Hypergeometric Overlap (RRHO)}

RRHO is a threshold free approach used to identify genome-wide expression patterns between two ranked gene lists. RRHO analysis was performed using the RRHO2 (v1.0) package with parameters of stepsize $=100$ and boundary $=0.02$. First, transcripts from each DESeq comparison are ranked by their p-value and effect size direction:

$$
\text { rankValue }=-1 * \log _{10}(\text { pvalue }) * \operatorname{sign}\left(\log _{2} \text { FoldChange }\right)
$$

This results in a ranked list where the most significantly upregulated genes are at the top of the list and the most significantly downregulated genes are at the bottom of the list with each gene appearing only once. Next, two ranked gene lists are compared to each other using a hypergeometric distribution to determine the significance of the number of overlapping genes within defined sample populations. Sample populations are independently defined for each list. Each population consists of all of the genes that are ranked higher than a specified threshold. Thresholds successively move down a list at a user specified step size. For computation convenience, here we define our step size as 100 genes. However, the smallest possible step size is 1 gene and the largest possible step size is the number of genes found in the smallest list. The hypergeometric $\mathrm{p}$-values resulting from comparing the List 1 genes (all genes above threshold $x$ ) to List 2 (all genes above threshold y) then populate a square matrix. As a result, the furthest bottom-left position of the matrix is the p-value of the overlap between genes ranked above the 1st threshold in each list and the furthest top-right position is the overlap between genes ranked above the last threshold in each list. As another example, the furthest bottom-right position is the $\mathrm{p}$-value comparing all genes above the last threshold of List 1 to all genes above the 1st threshold of List 2. The final matrix of hypergeometric p-values is visualized as a heatmap where List 1 is along the $x$-axis and List 2 is along the $y$-axis and each point represents a pvalue. The axises are arranged such that genes that are upregulated in both lists are in the bottom-left quadrant (quadrant UU) and genes that are downregulated in both lists are in the top-right quadrant (quadrant DD). Genes with opposite regulation in each condition, up in one list but down in the other, are along the opposite diagonal (quadrants UD and DU) (Fig.ure 2E). To visualize RRHO our heatmaps with a consistent $\mathrm{p}$-value scale we set the scale maximum $=300$ and the minimum $=1$.

The genes in each quadrant were extracted to form UU, DD, $\mathrm{UD}$, and DU gene lists for further analysis. Using these gene lists, the significant overlap between quadrants were determined using the SuperExactTest package. Gene ontology and pathway analysis of these gene lists were performed using the $\mathrm{R}$ package enrichGO with $M u s$ musculus ontology terms. Representative terms with an adjusted $p$-value of $<0.05$ were hand selected.

Additional information for RRHOs are in Fig. S2. Unscaled p-value RRHOs can be found in Fig. S2A-C. Since the pvalue of the hypergeometirc distribution is sensitive to the number of genes, RRHO also plots a log-Odds ratio for all comparisons. Log-Odds ratio plots can be found in Fig. S2D-F where the maximum scale was changed from 8 to 6 on all plots to better visualize values. Finally, to ensure that the signal we see in the RRHOs is not due to chance, we shuffled the ranked order of List 1 in each RRHO (Fig. S2GI). The resulting lack of signal throughout the RRHOs support that the signal we see in our experimental RRHOs is not due to chance.

\section{Pathway and Upstream Regulator Analysis with Ingenuity Pathway Analysis (IPA)}

Ingenuity pathway analysis (Qiagen, Germantown, MD), was used to determine potential biological pathways and upstream regulators of DEGs and co-regulated genes identified using RRHO. Core expression analysis was applied for each gene list followed by comparison analysis for related lists to help identify common pathways and regulators across groups. For both, outcomes were filtered to include only those that reached an activation z-score of $+/-2$ and a corrected p-value of $<0.05$ (corrected by Benjamini Hochberg). All predicted biological pathways 
Sadino et al., 13 July 2021-BioRxiv

and upstream regulators are presented in Supplemental Table 3.

\section{Weighted Correlation Network Analysis (WGCNA)}

A weighted gene co-expression network analysis was conducted using the R package WGCNA (v1.70-3) (47). WGCNA analysis was done using the Input and Pulldown sequencing data from only animals in the vTRAP cohorts (opposite-sex remain paired 4 weeks, opposite-sex separated 4 weeks, and same-sex separated 4 weeks). A signed co-expression network was constructed using a power of 9 and minimum module size of 30 genes according to the author's standard module-trait relationship workflow. No samples were designated as outliers using complete clustering. Gene modules were then correlated to either the trait Input or Pulldown to determine the gene clusters associated with each sample type. To determine which correlation direction (positive or negative) is associated with which sample type, the significantly positively or negatively correlated modules were grouped and analyzed by Enrichr for cell types (49-51). The top term for the positively correlated modules was nucleus accumbens while the top term for the negatively correlated modules was spinal cord. Therefore, the positively correlated modules represent the Pulldown samples, and by extension, neuronally enriched genes.

\section{Neuronal enriched genes throughout separation}

To robustly define neuronally enriched genes we found the intersection of the vTRAP Input vs Pulldown enriched DEGs (Fig. 4B) and the genes from the significantly, positively correlated WGCNA modules (Fig. 4D). The resulting intersection-2,365 genes - is referred to as "neuronal enriched genes" and includes almost all of the vTRAP enriched DEGs. To further validate the neuronal enriched genes we analyzed this gene list using Enrichr for cell types. The top two terms are nucleus accumbens and dorsal striatum confirming the specificity of this gene list.

We next wanted to follow the expression of these neuronal enriched genes throughout separation. First, since we only had vTRAP samples from specific cohorts in our experiment, we ensured that neuronal gene expression can be inferred from bulk sequencing of the same genes. To validate this, we filtered the Input and Pulldown samples of the opposite-sex 4 week separated cohort and compared the expression of the neuronal enriched genes using
Morpheus (Fig. S5C). The strong similarity in expression of the neuronal enriched genes using two different collection methods validated that bulk sequencing expression can be used to infer the neuron specific expression of those same genes. Second, to compare the expression patterns of the neuronal enriched genes to the bulk sequencing data we filtered the bulk data for only the neuronal genes. We then clustered the unfiltered bulk sequencing data, from $3 F$, and the neuronal enriched genes only bulk sequencing data using one minus Pearson correlation with complete linkage. For each neuronal cluster we then found the corresponding cluster in the unfiltered expression data with the correspondence links visualized by a Sankey plot using the $\mathrm{R} \quad$ package networkD3 (v0.4) (http://christophergandrud.github.io/networkD3/). Third, we identified three clusters in the neuronal enriched data that had differential expression based on separation duration. We analyzed each gene list using Gene Ontology as previously described and highlighted specific genes associated with top terms.

\section{Author Contributions and Notes}

Z.R.D. and J.M.S developed experimental design. J.M.S., C.J.K., X.G.B. executed experiments. J.M.S, D.M.W, and Z.R.D. analyzed and interpreted data. Z.R.D. and J.M.S. wrote the manuscript.

The authors declare no conflict of interest.

All sequencing data will be assigned a GEO accession number by the time of publication. All code will be available on the Donaldson Lab GitHub (https://github.com/donaldsonlab) and all additional data will be available on Figshare. The vole optimized DIOeGFP-RPL10a vector will be made available on Addgene.

\section{Acknowledgments}

We thank Robin Dowell, Mary Allen, Ryan Logan, Xiangning Xue, and Gracie Sapp for their assistance in designing the experiments and analysis. We also thank Cayla Jo Paulson and Jessica Abazaris of the animal care staff at the University of Colorado Boulder. We thank the rest of the Donaldson lab for their feedback and support and the voles for their sacrifice. For vTRAP images we used the Molecular, Cellular, and Developmental Biology Light 
bioRxiv preprint doi: https://doi.org/10.1101/2021.07.14.452355; this version posted July 14, 2021. The copyright holder for this preprint (which was not certified by peer review) is the author/funder, who has granted bioRxiv a license to display the preprint in perpetuity. It is made available under aCC-BY-NC-ND 4.0 International license.

Sadino et al., 13 July 2021 -BioRxiv

Microscopy Core at the University of Colorado Boulder with James Orth's assistance. This work was supported by $\mathrm{NIH}$ award DP2OD026143 and funds from the Whitehall Foundation and the Dana Foundation (to Z.R.D.) and NIH award T32 GM008759-17/18 (to J.M.S.).

\section{References}

1. K. M. Keyes, C. Pratt, S. Galea, K. A. McLaughlin, K. C. Koenen, M. K. Shear, The Burden of Loss: Unexpected death of a loved one and psychiatric disorders across the life course in a national study. Am J Psychiatry. 171, 864-871 (2014).

2. T. H. Holmes, R. H. Rahe, The social readjustment rating scale. Journal of Psychosomatic Research. 11, 213-218 (1967).

3. K. Shear, H. Shair, Attachment, loss, and complicated grief. Developmental Psychobiology. 47, 253-267 (2005).

4. T. D. Blocker, A. G. Ophir, A preference to bond? Male prairie voles form pair bonds even in the presence of multiple receptive females. Animal Behaviour. 122, 89-97 (2016).

5. S. Carter, Development of Partner Preferences in Female Prairie Voles ( Microtus ochrogaster ): The Role of Social and Sexual Experience. Hormones and Behavior. 349, 339-349 (1992).

6. L. L. Getz, C. S. Carter, L. Gavish, The mating system of the prairie vole, Microtus ochrogaster: Field and laboratory evidence for pairbonding. Behavioral Ecology and Sociobiology. 8, 189-194 (1981).

7. J. R. WILLIAMS, C. S. CARTER, T. INSEL, Partner Preference Development in Female Prairie Voles Is Facilitated by Mating or the Central Infusion of Oxytocin. Annals of the New York Academy of Sciences. 652, 487-489 (1992).

8. T. R. Insel, L. J. Young, Neurobiology of Attachment. Neuroscience. 2 (2001).

9. C. Sue Carter, A. Courtney Devries, L. L. Getz, Physiological substrates of mammalian monogamy: The prairie vole model. Neuroscience and Biobehavioral Reviews. 19, 303-314 (1995).

10. J. T. Winslow, N. Hastlngst, C. S. Cartert, C. R. Harbaugh, R. Thomas, A role for central vasopressin in pair bonding in monogamous prairie voles. Letters to Nature. 365, 545-548 (1993).

11. N. McNeal, M.-A. L. Scotti, J. Wardwell, D. L. Chandler, S. L. Bates, M. LaRocca, D. M. Trahanas, A. J. Grippo, Disruption of social bonds induces behavioral and physiological dysregulation in male and female prairie voles. Autonomic Neuroscience. 180, 9-16 (2014).

12. A. J. Grippo, B. S. Cushing, C. S. Carter, Depression-like behavior and stressor-induced neuroendocrine activation in female prairie voles exposed to chronic social isolation. Psychosomatic Medicine. 69, 149-157 (2007).

13. O. J. Bosch, J. Dabrowska, M. E. Modi, Z. V. Johnson, A. C. Keebaugh, C. E. Barrett, T. H. Ahern, J. Guo, V. Grinevich, D. G. Rainnie, I. D. Neumann, L. J. Young, Oxytocin in the nucleus accumbens shell reverses CRFR2-evoked passive stress-coping after partner loss in monogamous male prairie voles. Psychoneuroendocrinology. 64, 66-78 (2016).

14. T. T. Pohl, L. J. Young, O. J. Bosch, Lost connections: Oxytocin and the neural, physiological, and behavioral consequences of disrupted relationships. International Journal of Psychophysiology. 136, 54-63 (2019).

15. Y. Osako, R. Nobuhara, Y.-C. P. Arai, K. Tanaka, L. J. Young, M. Nishihara, S. Mitsui, K. Yuri, Partner Loss in Monogamous Rodents: Modulation of pain and emotional behavior in male prairie voles. Psychosomatic Medicine. 80, 62-68 (2018).

16. K. J. Harbert, M. Pellegrini, K. M. Gordon, Z. R. Donaldson, How prior pair-bonding experience affects future bonding behavior in monogamous prairie voles. Hormones and Behavior. 126, 104847 (2020).

17. N. S. Lee, N. L. Goodwin, K. E. Freitas, A. K. Beery, Affiliation, aggression, and selectivity of peer relationships in meadow and prairie voles. Frontiers in Behavioral Neuroscience. 13 (2019), doi:10.3389/fnbeh.2019.00052.

18. N. L. Goodwin, S. A. Lopez, N. S. Lee, A. K. Beery, Comparative role of reward in long-term peer and mate relationships in voles. Hormones and Behavior, 1-8 (2018).

19. N. S. Lee, A. K. Beery, The role of dopamine signaling in prairie vole peer relationships. Hormones and Behavior. 127, 104876-104876 (2021).

20. F. Duclot, L. Sailer, P. Koutakis, Z. Wang, M. Kabbaj, Transcriptomic Regulations Underlying Pair-bond Formation and Maintenance in the Socially Monogamous Male and Female Prairie Vole. Biological Psychiatry, 1-11 (2020).

21. J. A. Tripp, A. Berrio, L. A. McGraw, M. V. Matz, J. K. Davis, K. Inoue, J. W. Thomas, L. J. Young, S. M. Phelps, Comparative neurotranscriptomics reveal widespread species differences associated with bonding. BMC Genomics. 22, 399 (2021).

22. S. L. Resendez, P. C. Keyes, J. J. Day, C. Hambro, C. J. Austin, F. K. Maina, L. N. Eidson, K. A. Porter-Stransky, N. Nevárez, J. W. McLean, M. A. Kuhnmuench, A. Z. Murphy, T. A. Mathews, B. J. Aragona, Dopamine and opioid systems interact within the nucleus accumbens to maintain monogamous pair bonds. eLife. 5 (2016), doi:10.7554/elife.15325.

23. B. J. Aragona, Y. Liu, J. T. Curtis, F. K. Stephan, Z. Wang, A Critical Role for Nucleus Accumbens Dopamine in Partner-Preference Formation in Male Prairie Voles. The Journal of Neuroscience. 23, 3483-3490 (2003).

24. K. L. Gobrogge, Y. Liu, L. J. Young, Z. Wang, Anterior hypothalamic vasopressin regulates pair-bonding and drug-induced aggression in a monogamous rodent. Proceedings of the National Academy of Sciences (2009).

25. K. E. Savell, J. J. Tuscher, M. E. Zipperly, C. G. Duke, R. A. Phillips, A. J. Bauman, S. Thukral, F. A. Sultan, N. A. Goska, L. lanov, J. J. Day, A dopamine-induced gene expression signature regulates neuronal function and cocaine response. Science Advances. 6, eaba4221 (2020).

26. S. D. Cardoso, M. C. Teles, R. F. Oliveira, Neurogenomic mechanisms of social plasticity. J Exp Biol. 218, 140-149 (2015).

27. A. Zayed, G. E. Robinson, Understanding the Relationship Between Brain Gene Expression and Social Behavior: Lessons from the Honey Bee. Annual Review of Genetics. 46, 591-615 (2012).

28. J. A. Tripp, N. Y. Feng, A. H. Bass, Behavioural tactic predicts preoptic-hypothalamic gene expression more strongly than developmental morph in fish with alternative reproductive tactics. Proceedings of the Royal Society B: Biological Sciences. 285, 20172742 (2018).

29. S. L. Resendez, M. Kuhnmuench, T. Krzywosinski, B. J. Aragona, kOpioid Receptors within the Nucleus Accumbens Shell Mediate Pair Bond Maintenance. Journal of Neuroscience. 32, 6771-6784 (2012).

30. B. J. Aragona, Y. Liu, Y. J. Yu, J. T. Curtis, J. M. Detwiler, T. R. Insel, Z. Wang, Nucleus accumbens dopamine differentially mediates the formation and maintenance of monogamous pair bonds. Nature Neuroscience. 9, 133-139 (2006).

31. M. I. Love, W. Huber, S. Anders, Moderated estimation of fold change and dispersion for RNA-seq data with DESeq2. Genome Biology. 15, 550-550 (2014).

32. D. M. Walker, H. M. Cates, Y.-H. E. Loh, I. Purushothaman, A. Ramakrishnan, K. M. Cahill, C. K. Lardner, A. Godino, H. G. Kronman, J. Rabkin, Z. S. Lorsch, P. Mews, M. A. Doyle, J. Feng, B. Labonté, J. W. Koo, R. C. Bagot, R. W. Logan, M. L. Seney, E. S. Calipari, L. Shen, E. J. Nestler, Cocaine Self-administration Alters 
bioRxiv preprint doi: https://doi.org/10.1101/2021.07.14.452355; this version posted July 14, 2021. The copyright holder for this preprint (which was not certified by peer review) is the author/funder, who has granted bioRxiv a license to display the preprint in perpetuity. It is made available under aCC-BY-NC-ND 4.0 International license.

Sadino et al., 13 July 2021—BioRxiv

Transcriptome-wide Responses in the Brain's Reward Circuitry. Biological psychiatry. 84, 867-880 (2018).

33. M. Wang, Y. Zhao, B. Zhang, Efficient Test and Visualization of Multi-Set Intersections. Scientific Reports. 5, 16923-16923 (2015).

34. S. B. Plaisier, R. Taschereau, J. A. Wong, T. G. Graeber, Rankrank hypergeometric overlap: identification of statistically significant overlap between gene-expression signatures. Nucleic Acids Research. 38, e169-e169 (2010).

35. K. M. Cahill, Z. Huo, G. C. Tseng, R. W. Logan, M. L. Seney, Improved identification of concordant and discordant gene expression signatures using an updated rank-rank hypergeometric overlap approach. Scientific Reports. 8, 1-11 (2018).

36. G. Yu, L.-G. Wang, Y. Han, Q.-Y. He, clusterProfiler: an R Package for Comparing Biological Themes Among Gene Clusters. OMICS. 16, 284-287 (2012).

37. M. K. Loth, Z. R. Donaldson, Oxytocin, Dopamine, and Opioid Interactions Underlying Pair Bonding: Highlighting a Potential Role for Microglia. Endocrinology. 162 (2021), doi:10.1210/endocr/bqaa223.

38. H. Walum, L. J. Young, The neural mechanisms and circuitry of the pair bond. Nature Reviews Neuroscience. 19, 643-654 (2018).

39. M. E. Modi, L. J. Young, D-cycloserine facilitates socially reinforced learning in an animal model relevant to autism spectrum disorders. Biol Psychiatry. 70, 298-304 (2011).

40. Z. V. Johnson, L. J. Young, Oxytocin and vasopressin neural networks: Implications for social behavioral diversity and translational neuroscience. Neuroscience \& Biobehavioral Reviews. 76, 87-98 (2017).

41. A. S. Smith, C. Lieberwirth, Z. Wang, Behavioral and physiological responses of female prairie voles (Microtus ochrogaster) to various stressful conditions. Stress. 16, 531-539 (2013).

42. O. J. Bosch, H. P. Nair, T. H. Ahern, I. D. Neumann, L. J. Young, The CRF System Mediates Increased Passive Stress-Coping Behavior Following the Loss of a Bonded Partner in a Monogamous Rodent. Neuropsychopharmacology. 34, 1406-1415 (2009).

43. A. S. Smith, Z. Wang, Hypothalamic oxytocin mediates social buffering of the stress response. Biol Psychiatry. 76, 281-288 (2014).

44. J. P. Burkett, E. Andari, Z. V. Johnson, D. C. Curry, F. B. M. de Waal, L. J. Young, Oxytocin-dependent consolation behavior in rodents. Science. 351, 375-378 (2016).

45. M. Heiman, R. Kulicke, R. J. Fenster, P. Greengard, N. Heintz, Cell type - specific mRNA purification by translating ribosome affinity purification ( TRAP ). Nature Protocols. 9, 1282-1291 (2014).

46. M. Heiman, A. Schaefer, S. Gong, J. D. Peterson, M. Day, K. E. Ramsey, M. Suárez-Fariñas, C. Schwarz, D. A. Stephan, D. J. Surmeier, P. Greengard, N. Heintz, A Translational Profiling Approach for the Molecular Characterization of CNS Cell Types. Cell. 135, 738-748 (2008).

47. P. Langfelder, S. Horvath, WGCNA: An R package for weighted correlation network analysis. BMC Bioinformatics. 9, 559-559 (2008).

48. K. K. Pitchers, K. S. Frohmader, V. Vialou, E. Mouzon, E. J. Nestler M. N. Lehman, L. M. Coolen, DeltaFosB in the nucleus accumbens is critical for reinforcing effects of sexual reward. Genes Brain Behav. 9, 831-840 (2010).

49. E. Y. Chen, C. M. Tan, Y. Kou, Q. Duan, Z. Wang, G. V. Meirelles, N. R. Clark, A. Ma'ayan, Enrichr: interactive and collaborative HTML5 gene list enrichment analysis tool. BMC Bioinformatics. 14, 128 (2013)

50. M. V. Kuleshov, M. R. Jones, A. D. Rouillard, N. F. Fernandez, Q. Duan, Z. Wang, S. Koplev, S. L. Jenkins, K. M. Jagodnik, A. Lachmann, M. G. McDermott, C. D. Monteiro, G. W. Gundersen, A.
Ma'ayan, Enrichr: a comprehensive gene set enrichment analysis web server 2016 update. Nucleic Acids Res. 44, W90-97 (2016).

51. Z. Xie, A. Bailey, M. V. Kuleshov, D. J. B. Clarke, J. E. Evangelista, S. L. Jenkins, A. Lachmann, M. L. Wojciechowicz, E. Kropiwnicki, K. M. Jagodnik, M. Jeon, A. Ma'ayan, Gene Set Knowledge Discovery with Enrichr. Current Protocols. 1, e90 (2021).

52. P. Gong, J. Liu, P. R. Blue, S. Li, X. Zhou, Serotonin receptor gene (HTR2A) T102C polymorphism modulates individuals' perspective taking ability and autistic-like traits. Front Hum Neurosci. 9, 575 (2015).

53. C. M. Durand, C. Betancur, T. M. Boeckers, J. Bockmann, P. Chaste, F. Fauchereau, G. Nygren, M. Rastam, I. C. Gillberg, H. Anckarsäter, E. Sponheim, H. Goubran-Botros, R. Delorme, N. Chabane, M.-C. Mouren-Simeoni, P. de Mas, E. Bieth, B. Rogé, D. Héron, L. Burglen, C. Gillberg, M. Leboyer, T. Bourgeron, Mutations in the gene encoding the synaptic scaffolding protein SHANK3 are associated with autism spectrum disorders. Nat Genet. 39, 25-27 (2007).

54. K. Inoue, J. P. Burkett, L. J. Young, Neuroanatomical distribution of $\mu$-opioid receptor mRNA and binding in monogamous prairie voles (Microtus ochrogaster) and non-monogamous meadow voles (Microtus pennsylvanicus). Neuroscience. 244, 122-133 (2013).

55. L. Stetzik, D. Ganshevsky, M. N. Lende, L. E. Roache, S. Musatov, B. S. Cushing, Inhibiting ERa expression in the medial amygdala increases prosocial behavior in male meadow voles (Microtus pennsylvanicus). Behav Brain Res. 351, 42-48 (2018).

56. C. S. Carter, A. M. Perkeybile, The Monogamy Paradox: What Do Love and Sex Have to Do With It? Front Ecol Evol. 6, 202 (2018).

57. K. Lei, B. S. Cushing, S. Musatov, S. Ogawa, K. M. Kramer, Estrogen receptor-alpha in the bed nucleus of the stria terminalis regulates social affiliation in male prairie voles (Microtus ochrogaster). PLoS One. 5, e8931 (2010).

58. B. S. Cushing, A. Perry, S. Musatov, S. Ogawa, E. Papademetriou, Estrogen receptors in the medial amygdala inhibit the expression of male prosocial behavior. J Neurosci. 28, 10399-10403 (2008).

59. R. G. Arzate-Mejía, Z. Lottenbach, V. Schindler, A. Jawaid, I. M. Mansuy, Long-Term Impact of Social Isolation and Molecular Underpinnings. Front. Genet. 11 (2020), doi:10.3389/fgene.2020.589621.

60. D. L. Wallace, M.-H. Han, D. L. Graham, T. A. Green, V. Vialou, S. D. Iñiguez, J.-L. Cao, A. Kirk, S. Chakravarty, A. Kumar, V. Krishnan, R. L. Neve, D. C. Cooper, C. A. Bolaños, M. Barrot, C. A. McClung, E. J. Nestler, CREB regulation of nucleus accumbens excitability mediates social isolation-induced behavioral deficits. Nat Neurosci. 12, 200-209 (2009).

61. A. S. Smith, S. K. Williams Avram, A. Cymerblit-Sabba, J. Song, W. S. Young, Targeted activation of the hippocampal CA2 area strongly enhances social memory. Mol Psychiatry. 21, 1137-1144 (2016).

62. J. H. Kogan, P. W. Frankland, A. J. Silva, Long-term memory underlying hippocampus-dependent social recognition in mice. Hippocampus. 10, 47-56 (2000).

63. T. Okuyama, T. Kitamura, D. S. Roy, S. Itohara, S. Tonegawa, Ventral CA1 neurons store social memory. 353 (2012), doi:10.1177/1469605312439972.

64. M. A. van der Kooij, C. Sandi, Social memories in rodents: Methods, mechanisms and modulation by stress. Neuroscience \& Biobehavioral Reviews. 36, 1763-1772 (2012).

65. P. Sun, A. S. Smith, K. Lei, Y. Liu, Z. Wang, Breaking bonds in male prairie vole: Long-term effects on emotional and social behavior, physiology, and neurochemistry. Behavioural Brain Research. 265, 22-31 (2014).

66. C. Lieberwirth, Z. Wang, The Social Environment and Neurogenesis in the Adult Mammalian Brain. Front Hum Neurosci. 6 (2012), doi:10.3389/fnhum.2012.00118. 
bioRxiv preprint doi: https://doi.org/10.1101/2021.07.14.452355; this version posted July 14, 2021. The copyright holder for this preprint (which was not certified by peer review) is the author/funder, who has granted bioRxiv a license to display the preprint in perpetuity. It is made available under aCC-BY-NC-ND 4.0 International license.

Sadino et al., 13 July 2021 -BioRxiv

67. J. J. Harris, R. Jolivet, D. Attwell, Synaptic Energy Use and Supply. Neuron. 75, 762-777 (2012).

68. A. M. Kopec, C. J. Smith, N. R. Ayre, S. C. Sweat, S. D. Bilbo, Microglial dopamine receptor elimination defines sex-specific nucleus accumbens development and social behavior in adolescent rats. Nat Commun. 9, 3769 (2018).

69. T. T. Pohl, O. Jung, B. Di Benedetto, L. J. Young, O. J. Bosch, Microglia react to partner loss in a sex- and brain site-specific manner in prairie voles. Brain, Behavior, and Immunity (2021), doi:10.1016/j.bbi.2021.05.026.

70. V. Bonnefil, K. Dietz, M. Amatruda, M. Wentling, A. V. Aubry, J. L. Dupree, G. Temple, H.-J. Park, N. S. Burghardt, P. Casaccia, J. Liu, Region-specific myelin differences define behavioral consequences of chronic social defeat stress in mice. eLife. 8 doi:10.7554/eLife.40855.

71. M. L. Lehmann, T. K. Weigel, A. G. Elkahloun, M. Herkenham, Chronic social defeat reduces myelination in the mouse medial prefrontal cortex. Scientific Reports. 7, 46548 (2017).

72. B. Zhou, Z. Zhu, B. R. Ransom, X. Tong, Oligodendrocyte lineage cells and depression. Molecular Psychiatry. 26, 103-117 (2021).

73. M. Makinodan, K. M. Rosen, S. Ito, G. Corfas, A Critical Period for Social Experience-Dependent Oligodendrocyte Maturation and Myelination. Science. 337, 1357-1360 (2012).

74. J. Liu, K. Dietz, J. M. DeLoyht, X. Pedre, D. Kelkar, J. Kaur, V. Vialou, M. K. Lobo, D. M. Dietz, E. J. Nestler, J. Dupree, P. Casaccia, Impaired adult myelination in the prefrontal cortex of socially isolated mice. Nature Neuroscience. 15, 1621-1623 (2012).

75. R. L. Young, M. H. Ferkin, N. F. Ockendon-Powell, V. N. Orr, S. M. Phelps, Á. Pogány, C. L. Richards-Zawacki, K. Summers, T. Székely, B. C. Trainor, A. O. Urrutia, G. Zachar, L. A. O'Connell, H. A. Hofmann, Conserved transcriptomic profiles underpin monogamy across vertebrates. Proceedings of the National Academy of Sciences of the United States of America. 116, 1331-1336 (2019).

76. S. Potretzke, A. E. Ryabinin, The Prairie Vole Model of Pair-Bonding and Its Sensitivity to Addictive Substances. Front Psychol. 10, 2477 (2019).

77. K. A. Young, Y. Liu, Z. Wang, The neurobiology of social attachment: A comparative approach to behavioral, neuroanatomical, and neurochemical studies. Comp Biochem Physiol C Toxicol Pharmacol. 148, 401-410 (2008).

78. C. Lieberwirth, Z. Wang, The neurobiology of pair bond formation, bond disruption, and social buffering. Current Opinion in Neurobiology. 40, 8-13 (2016).
79. B. J. Aragona, Z. Wang, The prairie vole (Microtus ochrogaster): An animal model for behavioral neuroendocrine research on pair bonding. ILAR Journal. 45, 35-45 (2004).

80. M. M. Lim, L. J. Young, Vasopressin-dependent neural circuits underlying pair bond formation in the monogamous prairie vole. Neuroscience. 125, 35-45 (2004).

81. K. Horie, K. Inoue, K. Nishimori, L. J. Young, Investigation of Oxtrexpressing Neurons Projecting to Nucleus Accumbens using Oxtrires-Cre Knock-in prairie Voles (Microtus ochrogaster) Neuroscience. 448, 312-324 (2020).

82. J. Dimidschstein, Q. Chen, R. Tremblay, S. Rogers, G. Saldi, L. Guo, C. Xu, R. Liu, C. Lu, J. Chu, M. Avery, S. Rashid, M. Baek, A. Jacob, G. Smith, D. Wilson, G. Kosche, I. Kruglikov, T. Rusielewicz, V. Kotak, T. Mowery, S. Anderson, E. Callaway, J. Dasen, D. Fitzpatrick, V. Fossati, M. Long, S. Noggle, J. Reynolds, D. Sanes, B. Rudy, G. Feng, G. Fishell, A viral strategy for targeting and manipulating interneurons across vertebrate species. Nat Neurosci. 19, 1743-1749 (2016).

83. T. H. Ahern, M. E. Modi, J. P. Burkett, L. J. Young, Evaluation of two automated metrics for analyzing partner preference tests. Journal of Neuroscience Methods. 182, 180-188 (2009).

84. A. M. Bolger, M. Lohse, B. Usadel, Trimmomatic: a flexible trimmer for Illumina sequence data. Bioinformatics. 30, 2114-2120 (2014).

85. D. Kim, B. Langmead, S. L. Salzberg, HISAT: a fast spliced aligner with low memory requirements. Nature Methods. 12, 357-357 (2015).

86. P. Danecek, J. K. Bonfield, J. Liddle, J. Marshall, V. Ohan, M. O. Pollard, A. Whitwham, T. Keane, S. A. McCarthy, R. M. Davies, H. $\mathrm{Li}$, Twelve years of SAMtools and BCFtools. GigaScience. 10 (2021), doi:10.1093/gigascience/giab008.

87. P. T. Pyl, S. Anders, W. Huber, HTSeq-a Python framework to work with high-throughput sequencing data. Bioinformatics. 31, 166169 (2014).

88. R: The R Project for Statistical Computing, (available at https://www.r-project.org/).

89. H. Wickham, ggplot2: Elegant Graphics for Data Analysis (Springer International Publishing, ed. 2, 2016; https://www.springer.com/gp/book/9783319242750), Use R!

90. K. Blighe, kevinblighe/EnhancedVolcano (2021; https://github.com/kevinblighe/EnhancedVolcano). 